\title{
Asymmetric Dark Matter Models in SO(10)
}

\author{
Natsumi Nagata ${ }^{1}$, Keith A. Olive ${ }^{2,3}$ and Jiaming Zheng $^{2}$ \\ ${ }^{1}$ Department of Physics, University of Tokyo, Bunkyo-ku, Tokyo 113-0033, Japan \\ ${ }^{2}$ School of Physics and Astronomy, University of Minnesota, Minneapolis, MN 55455, USA \\ ${ }^{3}$ William I. Fine Theoretical Physics Institute, School of Physics and Astronomy, \\ University of Minnesota, Minneapolis, MN 55455, USA
}

\begin{abstract}
We systematically study the possibilities for asymmetric dark matter in the context of non-supersymmetric $\mathrm{SO}(10)$ models of grand unification. Dark matter stability in $\mathrm{SO}(10)$ is guaranteed by a remnant $\mathbb{Z}_{2}$ symmetry which is preserved when the intermediate scale gauge subgroup of $\mathrm{SO}(10)$ is broken by a $\mathbf{1 2 6}$ dimensional representation. The asymmetry in the dark matter states is directly generated through the out-of-equilibrium decay of particles around the intermediate scale, or transferred from the baryon/lepton asymmetry generated in the Standard Model sector by leptogenesis. We systematically classify possible asymmetric dark matter candidates in terms of their quantum numbers, and derive the conditions for each case that the observed dark matter density is (mostly) explained by the asymmetry of dark matter particles.
\end{abstract}

November 2016 


\section{Introduction}

Using only six parameters, the so-called concordance model of standard Big Bang cosmology does remarkably well in describing the observed Universe as seen for example in recent cosmic microwave background (CMB) [1], supernovae [2], and baryon acoustic oscillation (BAO) [3] data. Among these parameters are the density of baryons, $\Omega_{B} h^{2}=0.022$, and the density of cold dark matter, $\Omega_{c} h^{2}=0.12$, where quoted values are taken from Ref. [1]. Most studies attempting to explain the density of dark matter assume thermal initial conditions and compute the relic density remaining after the freeze-out of primordial annihilations. The thermal freeze-out mechanism was first used to set constraints on (then) possible heavy neutrino masses [4, 6]. Updated calculations [7,8] for heavy neutrino dark matter candidates provided lower bounds of 3-7 GeV depending on whether the neutrinos had Dirac or Majorana masses and details of the quark hadron transition in the early Universe [8,9].

Asymmetric dark matter first emerged as a means for complementing the above limits on neutrino masses [10]. For the case of Dirac neutrino masses, an asymmetry between neutrinos and anti-neutrinos allows one to place an upper limit on the neutrino mass. If the asymmetry is of order the baryon asymmetry this upper limit is approximately $60 \mathrm{GeV}$, and it was demonstrated in Ref. [10] that the out-of-equilibrium decay scenario (the leading mechanism for generating a baryon asymmetry at the time) would indeed provide an asymmetry in a fourth generation neutrino comparable to the baryon asymmetry. The possible connection between the relatively similar baryon and dark matter densities has since been a motivating factor for current models of asymmetric dark matter $11-16$ (for reviews of asymmetric dark matter, see Ref. [17]).

While Standard Model (SM)-like neutrinos are no longer viable dark matter candidates, there are many other possibilities for asymmetric dark matter. Here, we consider the possibility for asymmetric dark matter in the context of non-supersymmetric $\mathrm{SO}(10)$ models of grand unification [18 28] (GUTs). In SO(10) models with an intermediate scale broken by a 126 dimensional representation of $\mathrm{SO}(10)$, a discrete $\mathbb{Z}_{2}$ symmetry is preserved at low energies 29]. In this case, a scalar belonging to either a $\mathbf{1 6}$ or $\mathbf{1 4 4}$ will be stable if it is the lightest member of the multiplet. In addition, these models may allow for the unification of the gauge couplings at the GUT scale [30, 31, with a sufficiently long proton lifetime and acceptably low neutrino masses [23. It has also been shown that models with scalar singlet dark matter protect the Higgs quartic coupling from running negative, thus preserving the stability of the electroweak vacuum, and can trigger 
radiative electroweak symmetry breaking at the weak scale [27].

In this work, we consider the possibility that the $\mathrm{SO}(10)$ dark matter candidate possesses an asymmetry which accounts for the observed relic density. The asymmetry in the dark matter states can be produced via the out-of-equilibrium decay of intermediate-scale particles, or by transferring a part of the asymmetry in the SM sector, which is generated by leptogenesis [32], to the dark matter sector. Preservation of this asymmetry in the background of sphaleron interactions, and possible dark matter-anti matter oscillations will impose stringent constraints on the possible models.

We show that in models with a minimal field content below the GUT scale, only scalar singlet dark matter models can be viable. Other models either do not allow for phenomenologically acceptable gauge coupling unification or are hypercharged and require mass splittings which are incompatible with other derived constraints. For the scalar singlet case, we show that an asymmetry produced by a transfer process requires a relatively low intermediate scale which is not possible in minimal models. In contrast, by including a fermionic singlet, cogenesis models which produce the dark matter asymmetry directly through out-of-equilibrium decay are possible.

We also consider some next-to-minimal models which contain additional fields. While the dark matter candidate itself is not asymmetric (it is in fact a Majorana fermion in both cases considered), its relic density is determined by an asymmetry produced by either cogenesis or transfer into one of the the additional states which subsequently decays to the dark matter candidate.

In the ensuing discussion, we will first briefly describe the $\mathrm{SO}(10)$ models under consideration. A generic argument for the generation of asymmetries in the dark matter, baryon, and lepton numbers in the $\mathrm{SO}(10)$ models is given in Sec. 2. We also discuss in Sec. 2 some generic problems associated with asymmetric hypercharged dark matter. In section 3, we concentrate on the possibility for asymmetric dark matter with scalar singlets. Here, we consider asymmetries produced by either thermal transfer or through cogenesis. To try expand the universe of asymmetric models, we consider some non-minimal models in section 4. Our conclusions will be given in section 5 . 


\section{Asymmetric dark matter in $\mathrm{SO}(10)$}

\section{$2.1 \mathrm{SO}(10)$ dark matter models}

As noted above, with the exception of the hierarchy problem, non-supersymmetric $\mathrm{SO}(10)$ GUT 30, 33 models may contain many of the features often used to motivate supersymmetric models of dark matter. The $\mathrm{SO}(10)$ models we consider here all feature an intermediate scale gauge subgroup of $\mathrm{SO}(10)$, which allows for the possibility for gauge coupling unification. The specific intermediate gauge group is determined by the vacuum expectation value (VEV) of the Higgs representation, $R_{1}$, breaking $\mathrm{SO}(10)$, which may be in a 45,54 or 210 representation. ${ }^{1}$

We employ a $\mathbf{1 2 6}$ to break the intermediate gauge group at the intermediate scale $M_{\text {int }}$ down to the $\mathrm{SM}$ in order to preserve a $\mathbb{Z}_{2}$ symmetry related to matter parity. The coupling of the $\mathbf{1 2 6}$ to SM matter fields embedded in a 16 representation of $\mathrm{SO}(10)$ naturally gives rise to a Majorana mass to the singlet component of the 16, $\nu_{R}^{c}$, of order $\langle\mathbf{1 2 6}\rangle \sim M_{\text {int }}$, which when combined with the Dirac mass arising from the VEV of the SM Higgs (now residing in a 10-plet of $\mathrm{SO}(10)$ ) gives rise to the seesaw mechanism for light neutrino masses [34]. Furthermore, the out-of-equilibrium decay of the right-handed neutrinos may yield a lepton asymmetry (leptogenesis [32]), which is transferred into a baryon asymmetry through electroweak sphaleron effects [35, 36].

Possible choices for dark matter candidates were discussed in detail in Ref. [23]. Here we will restrict our attention to the candidates given in Table 1 , namely, those who have a non-zero $B-L$ or hypercharge. Here, each dark matter multiplet is specified by its spin (S for scalars and $\mathrm{F}$ for fermions), $\mathrm{SU}(2)_{L}$ representation $\mathbf{n}$, and hypercharge $Y$, where the $Q=T_{3}+Y=0$ component is the dark matter candidate. In general, a fermionic dark matter candidate should be parity even and belong to a 10, 45, 54, 120, 126, $\mathbf{2 1 0}$ or $\mathbf{2 1 0}$ ' representation, while scalar dark matter is parity odd and belongs to a $\mathbf{1 6}$ or 144 representation. Depending on the dark matter and Higgs representation chosen, renormalization group evolution of the gauge couplings can be used to determine the GUT scale, the intermediate scale, and the value of the GUT gauge coupling. We also note that the presence of the intermediate gauge symmetry, as well as dark matter fields which couple to the SM Higgs field, may prevent the Higgs quartic coupling from running negative at high energies. In fact, it has been shown 27] that for models in which the dark matter is a scalar singlet, the stability of the Higgs vacuum can be ensured and the

\footnotetext{
${ }^{1}$ In this paper, we restrict our attention to $\mathrm{SO}(10)$ irreducible representations with dimensions up to 210.
} 
Table 1: Partial list of $S U(2)_{L} \otimes U(1)_{Y}$ multiplets in $S O(10)$ representations that contain an electric neutral color singlet.

\begin{tabular}{lccll}
\hline \hline Model & $B-L$ & $\mathrm{SU}(2)_{L}$ & $Y$ & $\mathrm{SO}(10)$ representations \\
\hline $\mathrm{S}_{\mathbf{1}}^{0}$ & 1 & $\mathbf{1}$ & 0 & $\mathbf{1 6}, \mathbf{1 4 4}$ \\
$\mathrm{S}_{\mathbf{2}}^{1 / 2}$ & 1 & $\mathbf{2}$ & $1 / 2$ & $\mathbf{1 6}, \mathbf{1 4 4}$ \\
$\mathrm{S}_{\mathbf{3}}^{0}$ & 1 & $\mathbf{3}$ & 0 & $\mathbf{1 4 4}$ \\
$\mathrm{S}_{\mathbf{3}}^{1}$ & 1 & $\mathbf{3}$ & 1 & $\mathbf{1 4 4}$ \\
\hline $\mathrm{F}_{\mathbf{2}}^{1 / 2}$ & 0 & $\mathbf{2}$ & $1 / 2$ & $\mathbf{1 0}, \mathbf{1 2 0}, \mathbf{1 2 6} \mathbf{2 1 0}^{\prime}$ \\
$\mathrm{F}_{\mathbf{3}}^{1}$ & 0 & $\mathbf{3}$ & 1 & $\mathbf{5 4}$ \\
$\mathrm{F}_{\mathbf{4}}^{1 / 2}$ & 0 & $\mathbf{4}$ & $1 / 2$ & $\mathbf{2 1 0}$ \\
$\mathrm{F}_{\mathbf{4}}^{3 / 2}$ & 0 & $\mathbf{4}$ & $3 / 2$ & $\mathbf{2 1 0}$ \\
$\widehat{\mathrm{F}}_{\mathbf{1}}^{0}$ & 2 & $\mathbf{1}$ & 0 & $\mathbf{1 2 6}$ \\
$\widehat{\mathrm{F}}_{\mathbf{2}}^{1 / 2}$ & 2 & $\mathbf{2}$ & $1 / 2$ & $\mathbf{2 1 0}$ \\
$\widehat{\mathrm{F}}_{\mathbf{3}}^{1}$ & 2 & $\mathbf{3}$ & 1 & $\mathbf{1 2 6}$ \\
\hline \hline
\end{tabular}

electroweak symmetry may be broken radiatively as in supersymmetric models.

\subsection{Generation of asymmetries}

In the $\mathrm{SO}(10)$ GUT models we consider in this paper, the $B-L$ symmetry is spontaneously broken at the intermediate scale $M_{\text {int }}$ by the VEV of a $\mathbf{1 2 6}$ field. At this scale, some of the components of a representation of the intermediate gauge symmetry acquire masses of $\mathcal{O}\left(M_{\text {int }}\right)$. For example, the singlet component of the $\mathbf{1 2 6}$ field, which sits inside a $(\overline{\mathbf{1 0}}, \mathbf{1}, \mathbf{3})$ if the intermediate gauge symmetry is $\mathrm{SU}(4)_{C} \otimes \mathrm{SU}(2)_{L} \otimes \mathrm{SU}(2)_{R}$, obtains a mass from its own VEV, just like the SM Higgs boson having a mass proportional to its VEV. The right-handed neutrinos are also such particles as they obtain masses from the $126 \mathrm{VEV}$ through their Yukawa couplings.

The decay of such intermediate-scale particles can generate a $B-L$ asymmetry. The $B-L$ charge in the decay process may not be conserved if the relevant diagrams contain the $126 \mathrm{VEV}$. C and CP invariance can also be violated if the vertices in the diagrams include $\mathrm{CP}$ phases. Thus, if this decay occurs out-of-equilibrium, a $B-L$ asymmetry can be generated. A well-known example is the generation of a lepton-number (and 
thus $B-L)$ asymmetry via the out-of-equilibrium decay of right-handed neutrinosleptogenesis [32, 37]. If there are no other $B-L$ violating processes in equilibrium, the generated $B-L$ asymmetry remains non-zero, which results in non-zero baryon and lepton asymmetries with the help of electroweak sphaleron processes [35, 36.

An asymmetry in the dark matter sector can also be generated through the production of the $B-L$ asymmetry. We can divide such generation mechanisms into two types. First, if intermediate-scale particles can decay not only into SM particles but also dark matter particles, their out-of-equilibrium decay can directly produce a $B-L$ asymmetry in the dark matter sector. If this $B-L$ asymmetry is not communicated to the SM sector in the thermal equilibrium, it remains in the dark matter sector independently, which results in an asymmetry in the dark matter number. The simultaneous generation of asymmetries in the dark matter, baryon, and lepton numbers is in fact considered in the original work [10], where heavy neutrino dark matter candidates were shown to have an asymmetry similar to the baryon asymmetry. More recently, this scenario has been dubbed cogenesis and has been discussed widely in the literature 38].

Second, even if the dark matter sector did not obtain an asymmetry at the outset, the $B-L$ asymmetry in the SM sector, which is generated via leptogenesis, may be transferred to the dark matter sector. For this to occur, some interactions that communicate the asymmetries between these sectors should be in thermal equilibrium after the leptogenesis. As we shall see below, in this case the thermalization conditions give strong constraints on dark matter models.

In the subsequent sections, we discuss each of these two scenarios.2 The first case is highly dependent on models as it relies on non-thermal processes at high energies. In the second case, on the other hand, there are some model-independent aspects, which we now discuss, that must be shared by all of our $\mathrm{SO}(10)$ dark matter candidates.

In the transfer scenario, some interactions which distribute $B-L$ between both the dark matter and SM sectors are required to be in thermal equilibrium. To derive constraints from this requirement, we first obtain the temperature associated with leptogenesis.

The tree-level decay rate for a heavy neutrino state $N_{i}$ (which are mainly right-handed neutrinos), with the Yukawa coupling matrix, $y$, to its left-handed counterpart and the

\footnotetext{
2 Actually, there is another type of scenario; an asymmetry is only produced in the dark matter sector, not in the SM sector, and the dark matter asymmetry is transmitted to the SM sector via some interactions so that the observed baryon asymmetry is realized [39]. In $\mathrm{SO}(10)$ GUTs, however, leptogenesis is formulated quite naturally (see, for instance, Ref. [40]), and thus we expect some $B-L$ asymmetry in the SM sector. For this reason, we do not argue this case in this paper.
} 
Higgs boson is given by

$$
\Gamma_{D_{i}}=\Gamma\left(N_{i} \rightarrow H+\ell\right)+\Gamma\left(N_{i} \rightarrow H^{*}+\bar{\ell}\right)=\frac{1}{8 \pi}\left(y y^{\dagger}\right)_{i i} M_{i}
$$

where $M_{i}$ are the masses of the heavy neutrino states.

If right-handed neutrinos are thermally produced in the early Universe and their decay occurs out-of-equilibrium, a maximal lepton asymmetry of order

$$
\frac{n_{L}}{s} \sim \frac{\epsilon}{g_{*}}
$$

is generated, where $n_{L}$ is the net lepton density, $s$ is the entropy density, $g_{*} \simeq 100$ is the number of degrees of freedom in the thermal plasma, and $\epsilon$ is a measure of the $\mathrm{C}$ and $\mathrm{CP}$ violation in the decay, which is given by

$$
\epsilon \simeq \frac{3}{16 \pi} \frac{1}{\left(y y^{\dagger}\right)_{11}} \sum_{i=2,3} \operatorname{Im}\left[\left(y y^{\dagger}\right)_{i 1}^{2}\right] \frac{M_{1}}{M_{i}},
$$

for $M_{1} \ll M_{2}, M_{3}$. Note that a non-zero $\epsilon$ is obtained only if we include loop decay processes with at least two generations of massive right-handed neutrinos. If, on the other hand, the right-handed neutrinos are produced at the end of inflation, the asymmetry can be related directly to the reheat temperature, $T_{R}$, by 41

$$
\frac{n_{L}}{s} \sim \epsilon \frac{n_{\nu_{R}}}{T_{R}^{3}} \sim \epsilon f \frac{n_{\eta}}{T_{R}^{3}} \sim \epsilon f \frac{T_{R}}{m_{\eta}},
$$

where $n_{\eta}$ is the number density of inflatons at the time of their decay, $f$ is the branching fraction into $\nu_{R}$, and $m_{\eta}$ is the inflaton mass. The produced lepton (or $B-L$ ) asymmetry then generates baryon asymmetry as we see in Sec. 2.3 .

In both of the cases, the produced right-handed neutrinos should decay out-of-equilibrium. In general, the condition for the out-of-equilibrium decay of $N_{i}$ is

$$
\alpha_{y_{i}} M_{P} \lesssim \mathcal{C} M_{i}
$$

where $\alpha_{y_{i}} \equiv\left(y y^{\dagger}\right)_{i i} / 8 \pi, M_{P}=1.2 \times 10^{19} \mathrm{GeV}$ is the Planck mass, and $\mathcal{C} \equiv\left(8 \pi^{3} g_{*} / 90\right)^{1 / 2} \simeq$ 16.6 assuming that the number of degrees of freedom is $g_{*} \simeq 100$. Clearly the time of decay, and hence the time of the generation of the lepton asymmetry, will be determined by the condition (5). Let us define the parameter, $\xi \equiv \alpha_{y_{1}} M_{P} / M_{1}$. One can show that there is a critical value for $\xi$ such that the right-handed neutrinos decay in either a matter or radiation dominated expansion

$$
\left\{\begin{array}{ll}
\xi \ll \xi_{c} & \text { Matter domination } \\
\xi \gg \xi_{c} & \text { Radiation domination }
\end{array},\right.
$$


where

$$
\xi_{c} \equiv \frac{2^{9 / 2}}{\mathcal{C}^{3} \pi^{2}} \zeta(3)^{2} \simeq 7 \times 10^{-4},
$$

with $\zeta(3) \simeq 1.2$. If the right-handed neutrinos come to dominate the energy density, they decay at a temperature

$$
T_{D}^{m}=\left(\frac{\pi}{4 \zeta(3)}\right)^{\frac{1}{3}} \xi^{\frac{2}{3}} M_{1} \simeq 0.87 \cdot \xi^{\frac{2}{3}} M_{1},
$$

and assuming their decay products thermalize instantaneously, the Universe reheats to

$$
T_{R}^{m}=\left(\frac{\xi}{\mathcal{C}}\right)^{\frac{1}{2}} M_{1}<\left(\frac{\xi_{c}}{\mathcal{C}}\right)^{\frac{1}{2}} M_{1} .
$$

If instead, the right-handed neutrinos do not dominate the energy density and decay in a radiation background, the decay temperature is given by

$$
T_{D}^{r}=\left(\frac{\xi}{\mathcal{C}}\right)^{\frac{1}{2}} M_{1} \lesssim M_{1}
$$

where we have used the condition (5). In this case, there is no appreciable reheating due to decay. For later use, we define $T_{B L}$ as the temperature at which the $B-L$ asymmetry was produced (corresponding to either the decay temperature for a radiation dominated decay, or the decay induced reheat temperature in a matter dominated decay) or the maximum temperature when sphalerons are in equilibrium whichever is lower. This can be regarded as the temperature when leptogenesis occurs.

\subsection{Thermal conditions for transfer and the dark matter mass}

The $B-L$ asymmetry generated by leptogenesis is transferred to the dark sector via effective operators of the form

$$
\mathcal{L}_{\text {eff }}=\frac{c_{d}}{\Lambda^{d-4}} \mathcal{O}_{\mathrm{DM}} \mathcal{O}_{\mathrm{SM}}+\text { h.c. }
$$

if they are in thermal equilibrium, where $\mathcal{O}_{\mathrm{DM}}$ is an operator which contains only the dark matter fields and has a non-zero dark-matter number while $\mathcal{O}_{\text {SM }}$ consists of SM fields only; $d \geq 4$ is the mass dimension of the operator; $\Lambda$ denotes the scale at which the effective operator is generated (in particular, $\Lambda=M_{\text {int }}$ in the models discussed below); $c_{d}$ is a dimension-less constant, which may involve additional suppression factors such as small 
Yukawa couplings. The necessary condition for the interaction induced by the operator to be in thermal equilibrium is then given by

$$
\left\{\begin{array}{ll}
T<T_{\mathrm{eq}} & \text { for } d=4 \\
T>T_{\mathrm{eq}} & \text { for } d \geq 5
\end{array},\right.
$$

where the decoupling temperature $T_{\text {eq }}$ is determined by the condition

$$
\left.\Gamma_{\mathcal{L}_{\mathrm{eff}}}\right|_{T_{\mathrm{eq}}} \simeq \frac{1}{8 \pi^{3}} \frac{c_{d}^{2}}{\Lambda^{2(d-4)}} T_{\mathrm{eq}}^{2(d-4)+1}=\left.\frac{\mathcal{C} T_{\mathrm{eq}}^{2}}{M_{P}} \simeq H\right|_{T_{\mathrm{eq}}}
$$

which gives

$$
T_{\text {eq }} \equiv \Lambda\left[\frac{8 \pi^{3} \mathcal{C} \Lambda}{c_{d}^{2} M_{P}}\right]^{\frac{1}{2(d-4)-1}} .
$$

Hence, for non-renormalizable operators, if $T_{\text {eq }}<T_{B L}$, there is a period during which they are in equilibrium. If the operator is renormalizable, then even though it is outof-equilibrium at $T=T_{B L}$, it will come into thermal equilibrium when the temperature becomes lower than $T_{\text {eq }}$.

The presence of such interactions in thermal equilibrium gives rise to a condition between the chemical potentials of SM fields and that of the dark matter field, which relates the $B-L$ asymmetry to the asymmetry in the dark-matter number. Let us derive this relation following the argument given in Ref. [12]. We focus on the dominant operator in Eq. (11), and assume that $\mathcal{O}_{\mathrm{DM}}$ contains $N_{\mathrm{DM}}$ dark matter fields (or, strictly speaking, the number of dark matter fields minus the number of anti-dark-matter fields) and $\mathcal{O}_{\mathrm{SM}}$ consists of $N_{Q}, N_{u_{R}}, N_{d_{R}}, N_{L}, N_{e_{R}}, N_{H}$ numbers of the left-handed quarks, right-handed up quarks, right-handed down quarks, left-handed leptons, right-handed charged leptons, and Higgs fields, respectively. The dark matter field is a $n_{\mathrm{DM}^{-}}$-dimensional representation of $\mathrm{SU}(2)_{L}$ and has the hypercharge $Y_{\mathrm{DM}}$ and $B-L$ charge $Q_{B-L}^{\mathrm{DM}}$. By assigning each particle species a chemical potential, and using gauge and Higgs interactions as conditions on these potentials one can write down a simple set of equations for various charge densities [42,43. Above the electroweak phase transition temperature, the conservation of the electroweak symmetry makes the chemical potential of the $W$ boson vanish: $\mu_{W}=0$. In equilibrium, the sphaleron process then yields the additional condition. $?^{3}$

$$
3 \mu_{u_{L}}+\mu_{\nu_{L}}=0
$$

\footnotetext{
${ }^{3}$ Here, we assume that the dark matter field is either a complex scalar or a Dirac fermion. In this case, the dark matter does not contribute to the condition 15 .
} 
where $\mu_{u_{L}}$ and $\mu_{\nu_{L}}$ are the chemical potentials for the left-handed up quark and lefthanded neutrino, respectively. The chemical equilibrium condition with respect to the interaction $\mathcal{L}_{\text {eff }}$ reads

$$
N_{\mathrm{DM}} \mu_{\mathrm{DM}}+\left(N_{Q}+N_{u_{R}}+N_{d_{R}}\right) \mu_{u_{L}}+\left(N_{L}+N_{e_{R}}\right) \mu_{\nu_{L}}+\left(N_{H}+N_{u_{R}}-N_{d_{R}}-N_{e_{R}}\right) \mu_{0}=0,
$$

where $\mu_{\mathrm{DM}}$ and $\mu_{0}$ are the chemical potentials for the dark matter and the Higgs field. In this paper, we focus on the case where the low-energy effective theory contains one $\mathrm{SU}(2)_{L}$ doublet Higgs boson; however, for one's convenience, in this section we keep the number of the Higgs doublets to be arbitrary and denote it by $n_{H}$, with the assumption that all of the Higgs fields have the same chemical potential $\mu_{0}$. In addition, since $\mathcal{L}_{\text {eff }}$ should be neutral under $\mathrm{U}(1)_{Y}$, we have

$$
Y_{\mathrm{DM}} N_{\mathrm{DM}}+\frac{1}{6} N_{Q}+\frac{2}{3} N_{u_{R}}-\frac{1}{3} N_{d_{R}}-\frac{1}{2} N_{L}-N_{e_{R}}+\frac{1}{2} N_{H}=0 .
$$

On the other hand, it is not necessary for the interaction $\mathcal{L}_{\text {eff }}$ to conserve $B-L$ as we will see below. Let us denote the entire $B-L$ charge of $\mathcal{L}_{\text {eff }}$ by $\Delta_{B-L}$.

$$
Q_{B-L}^{\mathrm{DM}} N_{\mathrm{DM}}+\frac{1}{3} N_{Q}+\frac{1}{3} N_{u_{R}}+\frac{1}{3} N_{d_{R}}-N_{L}-N_{e_{R}}=\Delta_{B-L} .
$$

By using Eqs. (15), (16), (17), and (18), we then obtain 4

$$
\mu_{\mathrm{DM}}=3 X_{\mathrm{DM}} \mu_{u_{L}}+\left(2 Y_{\mathrm{DM}}-X_{\mathrm{DM}}\right) \mu_{0}
$$

with

$$
X_{\mathrm{DM}} \equiv Q_{B-L}^{\mathrm{DM}}-\frac{\Delta_{B-L}}{N_{\mathrm{DM}}} .
$$

The electric charge density $Q$ in units of $T^{2} / 6$ is given by

$$
\begin{aligned}
Q & =6 \mu_{u_{L}}-6 \mu_{\nu_{L}}+\left(12+2 n_{H}\right) \mu_{0}+2 \mu_{\mathrm{DM}} k(z) \sum_{j=-J_{\mathrm{DM}}}^{J_{\mathrm{DM}}}\left(j+Y_{\mathrm{DM}}\right) \\
& =24 \mu_{u_{L}}+\left(12+2 n_{H}\right) \mu_{0}+2 \mu_{\mathrm{DM}} n_{\mathrm{DM}} Y_{\mathrm{DM}} k(z),
\end{aligned}
$$

${ }^{4}$ Note that Eqs. (17) and 18 read

$$
\begin{aligned}
& N_{Q}+N_{u_{R}}+N_{d_{R}}-3 N_{L}-3 N_{R}=3\left[\Delta_{B-L}-Q_{B-L}^{\mathrm{DM}} N_{\mathrm{DM}}\right], \\
& N_{u_{R}}-N_{d_{R}}-N_{e_{R}}+N_{H}=Q_{B-L}^{\mathrm{DM}} N_{\mathrm{DM}}-\Delta_{B-L}-2 Y_{\mathrm{DM}} N_{\mathrm{DM}},
\end{aligned}
$$

and Eqs. 15) and 16 give

$$
N_{\mathrm{DM}} \mu_{\mathrm{DM}}=-\left(N_{Q}+N_{u_{R}}+N_{d_{R}}-3 N_{L}-3 N_{e_{R}}\right) \mu_{u_{L}}-\left(N_{H}+N_{u_{R}}-N_{d_{R}}-N_{e_{R}}\right) \mu_{0} .
$$


where $J_{\mathrm{DM}} \equiv\left(n_{\mathrm{DM}}-1\right) / 2, z \equiv m_{\mathrm{DM}} / T$ with $m_{\mathrm{DM}}$ the dark matter mass, and

$$
k(z)= \begin{cases}\frac{3}{4 \pi^{2}} \int_{0}^{\infty} \frac{x^{2} d x}{\sinh ^{2}\left(\frac{\sqrt{x^{2}+z^{2}}}{2}\right)} & \text { for complex scalar } \\ \frac{3}{2 \pi^{2}} \int_{0}^{\infty} \frac{x^{2} d x}{\cosh ^{2}\left(\frac{\sqrt{x^{2}+z^{2}}}{2}\right)} & \text { for Dirac fermion }\end{cases}
$$

Note that $k(z) \rightarrow 1$ for $z \rightarrow 0$, while $k(z) \propto e^{-z}$ for $z \gg 1$. On the other hand, the dark matter multiplet does not give a contribution to the $\mathrm{SU}(2)_{L}$ charge $T_{3}$ due to $\operatorname{Tr}\left(T_{3}\right)=0$. By using Eqs. (22) and (24) with the condition $Q=0$, we can express $\mu_{\mathrm{DM}}$ in terms of $\mu_{u_{L}}$ :

$$
\mu_{\mathrm{DM}}=\frac{3\left[\left(10+n_{H}\right) X_{\mathrm{DM}}-8 Y_{\mathrm{DM}}\right]}{6+n_{H}+\left(2 Y_{\mathrm{DM}}-X_{\mathrm{DM}}\right) n_{\mathrm{DM}} Y_{\mathrm{DM}} k(z)} \mu_{u_{L}} .
$$

We can also express the $B-L$ charge density in terms of $\mu_{u_{L}}$. For later convenience, let us denote the contributions of the SM and dark matter particles to the $B-L$ charge density by $(B-L)_{\mathrm{SM}}$ and $(B-L)_{\mathrm{DM}}$, respectively, and obtain a relation between $(B-L)_{\mathrm{SM}}$ and the asymmetry in the dark matter sector. To that end, first we express $(B-L)_{\mathrm{SM}}$ in units of $T^{2} / 6$ in terms of $\mu_{u_{L}}$. By using Eq. (15), the condition $Q=0$, and Eq. (26), we have

$$
\begin{aligned}
(B-L)_{\mathrm{SM}} & =3\left(4 \mu_{u_{L}}-3 \mu_{\nu_{L}}+\mu_{0}\right) \\
& =\frac{3\left[13 n_{H}+66+2 n_{\mathrm{DM}} Y_{\mathrm{DM}} k(z)\left(13 Y_{\mathrm{DM}}-8 X_{\mathrm{DM}}\right)\right]}{6+n_{H}+\left(2 Y_{\mathrm{DM}}-X_{\mathrm{DM}}\right) n_{\mathrm{DM}} Y_{\mathrm{DM}} k(z)} \mu_{u_{L}} .
\end{aligned}
$$

Thus, the asymmetry in the dark matter sector in units of $T^{2} / 6, \Delta_{\mathrm{DM}}(z) \equiv 2 n_{\mathrm{DM}} k(z) \mu_{\mathrm{DM}} !^{5}$ is related to $(B-L)_{\mathrm{SM}}$ as

$$
\Delta_{\mathrm{DM}}(z)=\frac{2 n_{\mathrm{DM}} k(z)\left[\left(10+n_{H}\right) X_{\mathrm{DM}}-8 Y_{\mathrm{DM}}\right]}{13 n_{H}+66+2 n_{\mathrm{DM}} Y_{\mathrm{DM}} k(z)\left(13 Y_{\mathrm{DM}}-8 X_{\mathrm{DM}}\right)}(B-L)_{\mathrm{SM}} .
$$

This expression shows that the interaction $\mathcal{L}_{\text {eff }}$ should decouple at some point; otherwise, $\Delta_{\mathrm{DM}}(z)$ is suppressed due to the factor $k(z)$. For non-renormalizable interactions, the decoupling temperature $T_{\text {dec }}$ is equal to $T_{\text {eq }}$ if $T_{\text {eq }}>m_{\mathrm{DM}}$. If $T_{\text {eq }}<m_{\mathrm{DM}}$, we need to solve the Boltzmann equation to determine the decoupling temperature. For renormalizable interactions, once they are in thermal equilibrium, they decouple only below the dark matter mass (or other mass thresholds of particles participating in the interactions). Here, we assume that the interaction $\mathcal{L}_{\text {eff }}$ decouples before the electroweak phase transition. We may also consider the case where the interaction remains in equilibrium until the time of

\footnotetext{
${ }^{5}$ We include a factor of $n_{\mathrm{DM}}$ in the definition of $\Delta(\mathrm{DM})$ since all of the charged states in the dark matter multiplet decay into the neutral component in the end.
} 
the electroweak phase transition, or of sphaleron decoupling. After decoupling, the dark matter asymmetry freezes with a value of $\Delta_{\mathrm{DM}} \equiv \Delta_{\mathrm{DM}}\left(z_{\mathrm{dec}}\right)$ where $z_{\mathrm{eq}} \equiv m_{\mathrm{DM}} / T_{\mathrm{dec}}$.

We here note that even though the operator $\mathcal{L}_{\text {eff }}$ violates the $B-L$ symmetry, we can obtain a non-zero asymmetry in the dark matter number density. This is because there is a new conserved quantity instead of $B-L$, which makes asymmetries non-vanishing. To see this explicitly, let us define the dark matter number $D$ for which we assign $+1(-1)$ for a dark matter particle (anti-particle). The interaction (11) violates the conservation of the dark matter number by $N_{\mathrm{DM}}$ and $B-L$ by $\Delta_{B-L}$. However,

$$
X \equiv(B-L)-\frac{\Delta_{B-L}}{N_{\mathrm{DM}}} D
$$

is conserved by the interaction. This is the new conserved quantity which replaces $B-L 6$

$(B-L)_{\mathrm{SM}}$ in Eq. 28$)$ is related to the baryon and lepton asymmetries in the SM sector, $B_{\mathrm{SM}}$ and $L_{\mathrm{SM}}$, respectively, through the ordinary procedure [42, 43]. By using Eq. (15) and $Q=0$ with the dark matter contribution removed from Eq. (24), we can express $B_{\mathrm{SM}}$ and $L_{\mathrm{SM}}$ in terms of $\mu_{u_{L}}$ (in units of $T^{2} / 6$ ) as

$$
\begin{aligned}
& B_{\mathrm{SM}}=12 \mu_{u_{L}} \\
& L_{\mathrm{SM}}=9 \mu_{\nu_{L}}-3 \mu_{0}=-\frac{3\left(42+9 n_{H}\right)}{6+n_{H}} \mu_{u_{L}}
\end{aligned}
$$

if sphaleron processes decouple before the electroweak transition. In this case, the relation between $(B-L)_{\mathrm{SM}}$ and $B_{\mathrm{SM}}$ is given by

$$
B_{\mathrm{SM}}=\frac{4\left(6+n_{H}\right)}{66+13 n_{H}}(B-L)_{\mathrm{SM}} .
$$

Thus, in the absence of a $B-L$ asymmetry, there is no baryon asymmetry, and thus no dark matter asymmetry. If the sphaleron processes decouple after the electroweak transition [48], on the other hand, $\mu_{0}=0$ as the Higgs boson now develops a VEV, while now $\mu_{W}$ is non-vanishing. In this case, the electric charge is given by

$$
Q=6 \mu_{u_{L}}-6 \mu_{\nu_{L}}-2\left(8+n_{H}\right) \mu_{W}
$$

while the sphaleron condition reads

$$
3 \mu_{u_{L}}+2 \mu_{W}+\mu_{\nu_{L}}=0 .
$$

\footnotetext{
${ }^{6}$ Possibilities of generating non-zero baryon asymmetry in the presence of $(B-L)$-violating interactions in equilibrium are discussed in Ref. 43 47], where the theory possesses a conserved quantum number which replaces $B-L$, such as lepton flavor.
} 
Again, by imposing the electric neutrality $Q=0$, we can express $B_{\mathrm{SM}}$ and $L_{\mathrm{SM}}$ in terms of $\mu_{u_{L}}$ as

$$
\begin{aligned}
& B_{\mathrm{SM}}=12 \mu_{u_{L}}+6 \mu_{W}=\frac{12\left(8+n_{H}\right)}{2+n_{H}} \mu_{u_{L}}, \\
& L_{\mathrm{SM}}=9 \mu_{\nu_{L}}+6 \mu_{W}=-\frac{9\left(22+3 n_{H}\right)}{2+n_{H}} \mu_{u_{L}},
\end{aligned}
$$

so that

$$
B_{\mathrm{SM}}=\frac{4\left(8+n_{H}\right)}{98+13 n_{H}}(B-L)_{\mathrm{SM}} .
$$

Provided that the symmetric part of the dark matter sector is removed via annihilation, the present dark matter abundance is simply given by $\Delta_{\mathrm{DM}}$. Since it is related to $(B-$ $L)_{\mathrm{SM}}$, we can relate it to the baryon number density today via Eqs. (31) and (35). To explain the observed dark matter energy density, therefore, the dark matter mass should be

$$
m_{\mathrm{DM}}=m_{N}\left(\frac{\Omega_{c} h^{2}}{\Omega_{B} h^{2}}\right)\left|\frac{13 n_{H}+66+2 n_{\mathrm{DM}} Y_{\mathrm{DM}} k\left(z_{\mathrm{dec}}\right)\left(13 Y_{\mathrm{DM}}-8 X_{\mathrm{DM}}\right)}{2 n_{\mathrm{DM}} k\left(z_{\mathrm{dec}}\right)\left[\left(10+n_{H}\right) X_{\mathrm{DM}}-8 Y_{\mathrm{DM}}\right]}\right|\left[\frac{B_{\mathrm{SM}}}{(B-L)_{\mathrm{SM}}}\right],
$$

where $m_{N}$ is the nucleon mass..$^{7}$

The persistence of any $(B-L)$-violating interactions in conjunction with electroweak sphaleron effects could wipe out [49] both the baryon and lepton asymmetry. Such a interaction is described by a non-renormalizable operator which consists of only the SM fields (note that there is no renormalizable $(B-L)$-violating operator):

$$
\mathcal{L}_{\Delta(B-L)}=\frac{c_{\Delta(B-L)}}{\Lambda^{n}} \mathcal{O}_{\Delta(B-L)}
$$

where $n+4$ is the mass dimensions of the operator $\mathcal{O}_{\Delta(B-L)}$, and $c_{\Delta(B-L)}$ is a constant which may include an additional suppression factor. By requiring that this operator is out-of-equilibrium when leptogenesis occurs, we obtain the following condition:

$$
\frac{c_{\Delta(B-L)}}{\Lambda^{n}} \lesssim\left[\frac{8 \pi^{3} \mathcal{C} T_{B L}^{(1-2 n)}}{M_{P}}\right]^{\frac{1}{2}}
$$

In the case of leptogenesis, for example, the wash-out could occur through the $\Delta L=2$ effective operators of the form $y^{2} L L H H / M_{R}$, where $y$ and $M_{R}$ collectively denote the

\footnotetext{
${ }^{7}$ Let us compare our generic formulae with some results obtained in the previous studies. Thermal conditions for the $Y_{\mathrm{DM}}=0$ cases are presented in Ref. [12]. Our results are consistent with Eqs. (9) and (10) in the published version of Ref. 12 . By setting $n_{\mathrm{DM}}=2, n_{H}=1, X_{\mathrm{DM}}=0$, and $Y_{\mathrm{DM}}=1 / 2$, Eq. (28) reproduces Eq. (8) in Ref. 14 and Eq. (12) in Ref. 16] with appropriate changes of notation.
} 
neutrino Yukawa couplings and the right-handed masses, respectively. This corresponds to the case where $n=1, \Lambda=M_{R}$, and $c_{\Delta(B-L)}=y^{2}$. In equilibrium, this interaction adds the condition $\mu_{\nu_{L}}+\mu_{0}=0$ and hence drives all chemical potentials to 0 . The out-of-equilibrium condition for this operator is

$$
\Gamma_{\Delta L}=\frac{\zeta(3)}{8 \pi^{3}} \frac{y^{4} T^{3}}{M_{R}^{2}}<\frac{\mathcal{C} T^{2}}{M_{P}} \simeq H,
$$

yielding $42,49,51$

$$
\frac{M_{R}}{y^{2}} \gtrsim 0.017 \sqrt{T_{B L} M_{P}}
$$

where $H$ is the Hubble parameter. Similarly, it is possible to put constraints on other $B$ and/or $L$ violating operators [50] which include $R$-parity violating operators in supersymmetric models. In an inflationary context, if $M_{R}<m_{\eta}$ and the inflaton decays to right-handed neutrinos, then it is sufficient to satisfy the constraint

$$
\frac{M_{R}}{y^{2}} \gtrsim 0.017 \sqrt{T_{R} M_{P}} \text {. }
$$

\subsection{Hypercharged asymmetric dark matter}

As shown in Table 1, some of the dark matter candidates have a non-zero hypercharge. It is widely known that such hypercharged dark matter is severely restricted by direct detection experiments. Hypercharged dark matter can have a vector coupling with $Z$ boson, which induces a spin-independent scattering with a nucleon via $Z$-boson exchange. It turns out that its scattering cross section is larger than the current experimental limits by orders of magnitude.

This constraint can be evaded if there is some interaction which gives rise to a mass splitting between the dark matter particle and its antiparticle after electroweak symmetry breaking. In this case, a hypercharged Dirac fermion (complex scalar) splits into two Majorana fermions (real scalars). Since neither Majorana fermions nor real scalars can have a vector coupling, the above constraint can be evaded. If the mass splitting is smaller than $\sim 100 \mathrm{keV}$ [52,53], however, inelastic scattering via $Z$-boson exchange occurs, which is again stringently constrained by direct detection experiments. For detailed discussions on hypercharged dark matter in $\mathrm{SO}(10)$ GUTs, see Ref. [23].

For a Dirac fermion $\psi$ with hypercharge $Y$, the following higher-dimensional operator can generate a mass splitting between $\psi$ and its charge conjugate $\psi^{c}$ :

$$
\mathcal{L}_{\Delta m}=\frac{c_{\Delta m}}{2 \Lambda^{(4 Y-1)}}\left(H^{*}\right)^{4 Y} \overline{\psi^{c}} \psi+\text { h.c. },
$$


where $H$ denotes the Higgs field, and we suppress the $\mathrm{SU}(2)_{L}$ indices. $c_{\Delta m}$ is a dimensionless constant that contains the Clebsch-Gordan coefficient (see Ref. [53] for a more explicit expression). Once the Higgs field develops a $\operatorname{VEV}\langle H\rangle=v / \sqrt{2}$, this yields a mass splitting

$$
\Delta m=\frac{c_{\Delta m} v^{4 Y}}{2^{(2 Y-1)} \Lambda^{(4 Y-1)}} .
$$

By requiring $\Delta m \gtrsim 100 \mathrm{keV}$ to evade the direct detection limits, we obtain $\Lambda \lesssim 10^{9} \mathrm{GeV}$, $3 \times 10^{4} \mathrm{GeV}$, and $4 \times 10^{3} \mathrm{GeV}$ for $Y=\frac{1}{2}, 1, \frac{3}{2}$, respectively, where we set $c_{\Delta m}$ equal to the Clebsch-Gordan coefficient for the dark matter component. Similarly, for scalar dark matter with hypercharge $Y \geq 1$, the mass splitting can be induced by non-renormalizable operators generated at a high-energy scale $\Lambda$. For $m_{\mathrm{DM}}=1 \mathrm{TeV}$, the requirement of $\Delta m \gtrsim 100 \mathrm{keV}$ leads to $\Lambda \lesssim 10^{5} \mathrm{GeV}$ and $4 \times 10^{3} \mathrm{GeV}$ for $Y=1$ and $Y=\frac{3}{2}$, respectively. For the $Y=\frac{1}{2}$ case, on the other hand, the mass splitting is provided by a renormalizable operator, thus there is no limit on the high-energy scale.

The operator (42) has the form (11), and thus can communicate asymmetry in the SM sector to the dark matter sector [13, 14]. Hence, hypercharged dark matter can be a good candidate for asymmetric dark matter, and this possibility has been discussed in the literature [13, 15, 16]. As it turns out, however, there are two challenges in this scenario, besides the direct detection bound mentioned above. First, if the operator (42) remains in thermal equilibrium below the electroweak phase transition temperature, then it washes out the dark matter asymmetry. The chemical equilibrium condition for this interaction gives an additional relation between the dark matter and Higgs chemical potentials: $4 Y \mu_{0}+2 \mu_{\mathrm{DM}}=0$. After electroweak symmetry breaking, $\mu_{0}=0$, and thus this condition implies $\mu_{\mathrm{DM}}=0$. To avoid this, the interaction 42 should decouple before electroweak symmetry breaking. Second, the operator (42) causes particle-antiparticle oscillations after electroweak symmetry breaking, which may wash out the asymmetry in the dark sector. To prevent this, we need to make the oscillation rate sufficiently small or assure the decoupling of dark matter from thermal bath before the electroweak phase transition. In the latter case, there is no asymmetry in the dark matter sector at present, but still the dark matter abundance is (mainly) determined by the asymmetry of dark matter before the electroweak symmetry breaking.

If there were no limits from direct detection experiments on the interaction (42), then we could evade these problems by taking $\Lambda$ to be sufficiently high or the coefficient $c_{\Delta m}$ to be very small so that the decoupling of the interaction 42 is well above the electroweak transition and the particle-antiparticle oscillation induced due to the mass splitting $\Delta m$ is slow enough. As we will see below, however, these problems and the direct 
detection bound can be evaded simultaneously only if the dark matter mass is large enough compared with the electroweak phase transition temperature. On the other hand, we will see in the following discussion that in this case the annihilation of the symmetric part of dark matter tends to be insufficient so that the dark matter relic abundance exceeds the observed dark matter density. This incompatibility disfavors most of the hypercharged asymmetric dark matter candidates [13, 15, 16].

To see this, we give a rough estimate for the above conditions. First, according to Eq. (14), $T_{\text {eq }}$ for the operator 42 is given by

$$
T_{\mathrm{eq}}=\left[\frac{8 \pi^{3} \mathcal{C} \Lambda^{2(4 Y-1)}}{c_{\Delta m}^{2} M_{P}}\right]^{\frac{1}{2(4 Y-1)-1}}=\left[\frac{8 \pi^{3} \mathcal{C} v^{8 Y}}{4^{(2 Y-1)} M_{P} \Delta m^{2}}\right]^{\frac{1}{2(4 Y-1)-1}} .
$$

For $Y=1 / 2$, for instance, this reads

$$
T_{\text {eq }} \simeq 100 \mathrm{GeV} \times\left(\frac{100 \mathrm{keV}}{\Delta m}\right)^{2} .
$$

This result shows that the requirement $\Delta m \gtrsim 100 \mathrm{keV}$ to evade the direct detection bound may cause the operator (42) to remain in equilibrium down to the electroweak phase transition. We however note that the formula (14) is based on the assumption that all of the relevant particles are relativistic. Thus, if the dark matter mass is much larger than the electroweak scale, the above consequence may be modified significantly.

The second condition follows from $\Gamma_{\mathrm{osc}}<\left.H\right|_{T_{\mathrm{EW}}}$ where $\Gamma_{\mathrm{osc}}=\Delta m / 2$ is the rate of particle-antiparticle oscillations and $T_{\mathrm{EW}}$ is the temperature at the electroweak phase transition. This leads to

$$
\Delta m<\frac{2 \mathcal{C} T_{\mathrm{EW}}^{2}}{M_{P}} \simeq 3 \times 10^{-14} \mathrm{GeV} \times\left(\frac{T_{\mathrm{EW}}}{100 \mathrm{GeV}}\right)^{2} .
$$

Obviously, this conflicts with the direct detection bound. Thus, to avoid particle-antiparticle oscillations from erasing the dark matter asymmetry, the dark matter should decouple from thermal bath above $T_{\mathrm{EW}}$. Since the freeze-out temperature of dark matter is given by $\simeq m_{\mathrm{DM}} / 25$, this condition requires $m_{\mathrm{DM}} \gtrsim 25 T_{\mathrm{EW}}$.

As we have just seen, the above conditions may be evaded if $m_{\mathrm{DM}} \gg T_{\mathrm{EW}}$. On the other hand, there is an upper bound on the dark matter mass which follows from the requirement that the symmetric part of dark matter be annihilated away so that the asymmetric part accounts for the (dominant part of the) dark matter abundance. For example, for the $\mathrm{SU}(2)_{L}$ doublet $Y=1 / 2$ Dirac dark matter, the annihilation is effective if $m_{\mathrm{DM}}<1 \mathrm{TeV}$ [54]. On the other hand, the second condition discussed above requires 
$m_{\mathrm{DM}} \gtrsim 25 T_{\mathrm{EW}}>1 \mathrm{TeV}$, and thus the doublet Dirac fermion is unable to be asymmetric dark matter [13]. For the $\mathrm{SU}(2)_{L}$ doublet scalar dark matter, the upper bound on the dark matter mass is relaxed if the dark matter-Higgs quartic coupling is large. Even in this case, however, the dark matter asymmetry is found to be much smaller than the observed dark matter density once the perturbativity condition is imposed on the quartic coupling [16]. Other cases for hypercharged dark matter candidates are discussed in Ref. [15], and found that the $Y>1$ cases are excluded. As a consequence, only the $Y=1$ cases can be promising candidates for hypercharged asymmetric dark matter.

\subsection{Candidate models for $\mathrm{SO}(10)$ asymmetric dark matter}

Let us summarize the discussion in this section, and list up promising candidates for asymmetric dark matter in SO(10) GUTs. First, we consider the "minimal models", namely, we require that besides the SM particles only the dark matter multiplet has a mass much lighter than the intermediate scale. In this case, the low-energy effective theory only contains the SM particles and the dark matter, and the relevant non-renormalizable operators are generated at the intermediate or GUT scale.

As discussed in the previous subsection, the $Y=1 / 2$ and $3 / 2$ candidates in Table 1 have already been excluded. In addition, the analysis in Ref. [23] shows that $\mathrm{S}_{\mathbf{3}}^{0}, \mathrm{~S}_{\mathbf{3}}^{1}, \mathrm{~F}_{\mathbf{3}}^{1}$, $\widehat{\mathrm{F}}_{1}^{0}, \widehat{\mathrm{F}}_{3}^{1}$ are not good candidates for $\mathrm{SO}(10)$ dark matter models. This is because none of these models are consistent with gauge coupling unification with reasonable values of $M_{\text {int }}$ and/or $M_{\mathrm{GUT}}$ with minimal field content. As a result, only $\mathrm{S}_{1}^{0}$ can be a promising candidate for $\mathrm{SO}(10)$ asymmetric dark matter. We will discuss this candidate in the subsequent section. Then, we discuss some next-to-minimal extensions in Sec. 4.

\section{Scalar Singlet Asymmetric Dark Matter}

As we discussed in the previous section, singlet scalar dark matter is the only candidate for asymmetric dark matter in $\mathrm{SO}(10)$ if we require the minimality. We discuss this possibility in this section. First, in Sec. 3.1, we summarize possible scalar singlet dark matter models in $\mathrm{SO}(10)$ following the discussion in Ref. [23]. In Sec. 3.2, we derive the condition that the symmetric part of the scalar singlet dark matter is sufficiently annihilated away, and discuss the current experimental constraints. In Sec. 3.3, we argue for the necessity of suppressing the particle-antiparticle oscillations, and show that we can actually evade the oscillations by taking some relevant Lagrangian terms to be very small. Then, in Sec. 3.4 . 
we discuss the case where the dark matter asymmetry is thermally transferred from the $B-L$ asymmetry in the SM sector. We demonstrate that this possibility does not work for the $\mathrm{SO}(10)$ dark matter candidates given in Sec. 3.1. Finally, we consider the cogenesis scenario in Sec. 3.5.

\subsection{Scalar singlet dark matter candidates}

As shown in Table 1, singlet scalar dark matter belongs to either a $\mathbf{1 6}$ or $\mathbf{1 4 4}$ of SO(10). Below the GUT scale, there are several possibilities for the dark matter multiplet according to different intermediate gauge groups. As shown in Ref. [23], among these possibilities, only three accommodate a sufficiently high GUT scale, which is required to evade the proton decay bound. These models are called $\mathrm{SA}_{422}, \mathrm{SA}_{3221}$, and $\mathrm{SA}_{3221 \mathrm{D}}$ in Ref. [23], where the intermediate gauge groups are $\mathrm{SU}(4)_{C} \otimes \mathrm{SU}(2)_{L} \otimes \mathrm{SU}(2)_{R}, \mathrm{SU}(3)_{C} \otimes \mathrm{SU}(2)_{L} \otimes \mathrm{SU}(2)_{R} \otimes$ $\mathrm{U}(1)_{B-L}$, and $\mathrm{SU}(3)_{C} \otimes \mathrm{SU}(2)_{L} \otimes \mathrm{SU}(2)_{R} \otimes \mathrm{U}(1)_{B-L} \otimes D$, respectively, with $D$ denoting the so-called $D$-parity [55]. In the case of $\mathrm{SA}_{422}$, we fine-tune the mass of the $(\mathbf{4}, \mathbf{1}, \mathbf{2})$ representation of $\mathrm{SU}(4)_{C} \otimes \mathrm{SU}(2)_{L} \otimes \mathrm{SU}(2)_{R}$ in the dark matter multiplet to be much lighter than the GUT scale, while the rest of the components remain around the GUT scale. After the intermediate gauge symmetry is broken, only the singlet complex scalar component of the $(\mathbf{4}, \mathbf{1}, \mathbf{2})$ has a mass around the $\mathrm{TeV}$ scale via another fine-tuning, while the other components remain with masses of $\mathcal{O}\left(M_{\text {int }}\right)$. By solving RGEs, we obtain $M_{\mathrm{GUT}}=2.1 \times 10^{16} \mathrm{GeV}$ and $M_{\mathrm{int}}=1.2 \times 10^{11} \mathrm{GeV}$, and the proton lifetime is computed to be $\tau\left(p \rightarrow e^{+} \pi^{0}\right)=6.4 \times 10^{36}$ yrs, where the $\mathrm{SO}(10)$ gauge boson mass is set equal to $M_{\mathrm{GUT}}$. Similarly, for $\mathrm{SA}_{3221}$, the $(\mathbf{1}, \mathbf{1}, \mathbf{2}, 1)$ component lies below the GUT scale. The GUT and intermediate scales are found to be $4.6 \times 10^{16} \mathrm{GeV}$ and $3.4 \times 10^{8} \mathrm{GeV}$, respectively, and the proton lifetime is $1.4 \times 10^{38} \mathrm{yrs}$. For $\mathrm{SA}_{3221 \mathrm{D}}$, in addition to the $(\mathbf{1}, \mathbf{1}, \mathbf{2}, 1)$, the $(\mathbf{1}, \mathbf{2}, \mathbf{1}, 1)$ component also lies around the intermediate scale due to $D$-parity. In this case, we have $M_{\mathrm{GUT}}=3.8 \times 10^{15} \mathrm{GeV}, M_{\mathrm{int}}=1.2 \times 10^{10} \mathrm{GeV}$, and $\tau\left(p \rightarrow e^{+} \pi^{0}\right)=6.7 \times 10^{33}$ yrs. We note that the $\mathrm{SO}(10)$ gauge boson masses can be different from $M_{\mathrm{GUT}}$ by an $\mathcal{O}(1)$ factor, and thus we expect an order of magnitude uncertainty in the computation of proton lifetimes. Taking this uncertainty into account, all of these models are consistent with the present proton decay bound $\tau\left(p \rightarrow e^{+} \pi^{0}\right)>1.6 \times 10^{34}$ yrs 56 .

To be specific, we focus on the $\mathrm{SA}_{3221}$ case in the following analysis, but similar discussions can also be applied to the other cases. 


\subsection{Annihilations and experimental limits}

In order for the asymmetric dark matter scenario to work, the symmetric part of dark matter should efficiently be eliminated. This requirement gives a lower bound on the annihilation rate of dark matter. The annihilation of the singlet dark matter in our model proceeds through the dark matter-Higgs quartic coupling:

$$
\mathcal{L}_{\text {int }}=-\lambda_{S H}|S|^{2}|H|^{2},
$$

where $S$ denotes the scalar singlet dark matter. For a dark matter mass smaller than the weak gauge boson masses, the dominant annihilation mode is $S S^{*} \rightarrow b \bar{b}$. For $m_{\mathrm{DM}}>$ few $\times 100 \mathrm{GeV}$, on the other hand, the dark matter particles annihilate into a pair of the SM Higgs bosons or weak gauge bosons. We compute the relic abundance of the symmetric part, $\Omega_{\text {sym. }} h^{2}$, using micrOMEGAS [57]. We need to require that $\Omega_{\text {sym. }} h^{2}$ is much smaller than the observed dark matter density, $\Omega_{\mathrm{sym} .} h^{2} \ll 0.12$ [1], in order for the asymmetric part to be the dominant component of the dark matter abundance. In Fig. 1, we show in the $m_{\mathrm{DM}^{-}} \lambda_{S H}$ parameter space the region where $\Omega_{\mathrm{sym} .} h^{2}>0.12$ in the gray shaded area, which is phenomenologically excluded. In addition, the black dotted line shows the parameter points where the symmetric part is $10 \%$ of the total dark matter density. The region above (below) the line predicts a smaller (larger) abundance for the symmetric

part. As we can see, $\lambda_{S H} \gtrsim 0.1$ is required to sufficiently remove the symmetric part, with the exception of the resonance region $m_{\mathrm{DM}} \simeq m_{h} / 2$ with $m_{h} \simeq 125 \mathrm{GeV}$ the mass of the Higgs boson [58].

If the mass of the dark matter singlet is smaller than $m_{h} / 2$, the SM Higgs boson can decay into a pair of the dark matter particles through the interaction (47). This decay mode is invisible at the LHC, and reduces the branching fractions of the other decay channels, which is severely restricted by the Higgs measurements at the LHC [59]. Currently, LHC experiments give an upper limit on the branching ratio of the Higgs invisible decay mode, $\mathrm{BR}(\mathrm{inv})<.0.23[60,61$. In our model, the branching ratio of the invisible decay is evaluated as

$$
\mathrm{BR}(\text { inv. })=\frac{\Gamma\left(h \rightarrow S S^{*}\right)}{\Gamma\left(h \rightarrow S S^{*}\right)+\Gamma_{\text {Higgs }}},
$$

where $\Gamma_{\text {Higgs }}=4.07 \times 10^{-3} \mathrm{GeV}$ is the total decay width of a $125 \mathrm{GeV}$ Higgs boson, and

$$
\Gamma\left(h \rightarrow S S^{*}\right)=\frac{\lambda_{S H}^{2} v^{2}}{16 \pi m_{h}} \sqrt{1-\frac{4 m_{\mathrm{DM}}^{2}}{m_{h}^{2}}} .
$$




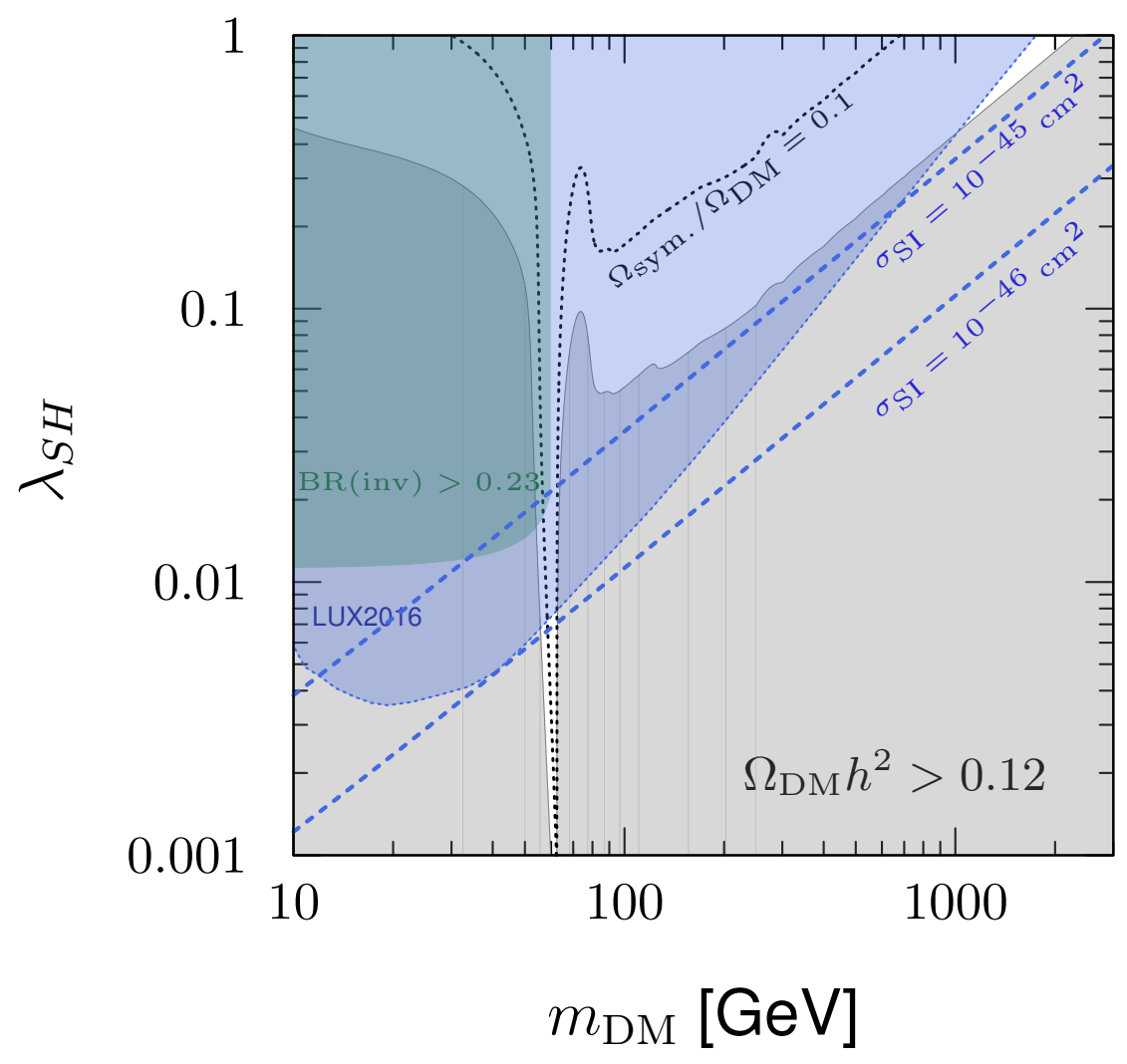

Figure 1: Constraints on the complex scalar singlet dark matter. The gray shaded area is excluded since the predicted dark matter abundance exceeds the observed value $\Omega_{\mathrm{DM}} h^{2}=$ 0.12 [1]. The green shaded region is excluded since the invisible decay branch of the Higgs boson is too large. The blue shaded region is excluded by the LUX 2016 result $\left[6^{7}\right]$. The black dotted line shows the parameter points where the symmetric part is $10 \%$ of the observed dark matter density. The upper (lower) blue dashed line corresponds to $\sigma_{\mathrm{SI}}^{(p)}=10^{-45} \mathrm{~cm}^{2}\left(10^{-46} \mathrm{~cm}^{2}\right)$.

Thus, the upper limit on BR(inv.) leads to an upper limit on $\lambda_{S H}$ for $m_{\mathrm{DM}}<m_{h} / 2$. We show the region excluded by the Higgs invisible decay bound in the green shaded region in Fig. 1. This indicates that $m_{\mathrm{DM}}<m_{h} / 2$ is excluded by a combination of this bound and the relic density of the symmetric part.

The interaction (47) also induces dark matter-nucleon scatterings via Higgs boson exchange, and thus direct detection experiments impose limits on the coupling $\lambda_{S H}{ }^{8}$ The spin-independent (SI) scattering cross section of dark matter with a nucleon $N(N=p$

\footnotetext{
${ }^{8}$ In the low dark matter mass region $m_{\mathrm{DM}} \lesssim 10 \mathrm{GeV}$, the dark matter-nucleon scattering cross section is also restricted by the existence of neutron stars 62 .
} 
or $n$ ) is computed as follows 63]:

$$
\sigma_{\mathrm{SI}}^{(N)}=\frac{f_{N}^{2} m_{N}^{2}}{4 \pi\left(m_{N}+m_{\mathrm{DM}}\right)^{2}}
$$

where $m_{N}$ is the nucleon mass and $f_{N}$ is given by

$$
\frac{f_{N}}{m_{N}}=\frac{\lambda_{S H}}{m_{h}^{2}}\left[\sum_{q=u, d, s} f_{T_{q}}^{(N)}+\frac{2}{9} f_{T G}^{(N)}\right],
$$

with the mass fractions $f_{T_{q}}^{(N)} \equiv\left\langle N\left|m_{q} \bar{q} q\right| N\right\rangle / m_{N}, m_{q}$ being the mass of a light quark $q$, and $f_{T G}^{(N)} \equiv 1-\sum_{q=u, d, s} f_{T_{q}}^{(N)}$. For the mass fractions we use the values computed by using lattice simulations in Ref. $|64|: f_{T_{u}}^{(p)}=0.0149, f_{T_{d}}^{(p)}=0.0234$, and $f_{T_{s}}^{(p)}=0.0440$. If we instead use the values obtained from the pion-nucleon $\sigma$-term $\Sigma_{\pi N}=50 \mathrm{MeV} 65$ and $\sigma_{0}=36 \mathrm{MeV}$ [66] following the discussion given in Ref. [65], we obtain a larger SI scattering cross section by a factor of $\simeq 2$. We show the contour of $\sigma_{\mathrm{SI}}^{(p)}=10^{-45} \mathrm{~cm}^{2}$ $\left(10^{-46} \mathrm{~cm}^{2}\right)$ by the upper (lower) blue dashed line in Fig. 1. We also show the current constraint from the LUX experiment [67] by the blue shaded area. We find that there are two allowed regions: $m_{\mathrm{DM}} \simeq m_{h} / 2$ and $\simeq 1 \mathrm{TeV}$. In the latter case, the symmetric part is the dominant component of the dark matter abundance. For a larger dark matter mass, a larger $\lambda_{S H}$ is required to eliminate the symmetric part of the dark matter abundance. However, $\lambda_{S H}$ cannot be too large; the requirement of perturbativity up to the GUT scale imposes an upper bound on the value of $\lambda_{S H}$ at the electroweak scale [27]. This upper bound depends on the particle content and other couplings in the model, but typically $\lambda_{S H} \lesssim 1$ [27]. According to Fig. 1, this indicates $m_{\mathrm{DM}} \lesssim 2 \mathrm{TeV}$. This dark matter mass region will soon be tested in dark matter direct detection experiments such as XENON1T [68]. In the former case, on the other hand, although it is possible for the asymmetric part to dominate the symmetric part, it is hard to probe the whole parameter space in future experiments due to the fact that $\lambda_{S H}$ must be small [69].

\subsection{Particle-antiparticle oscillations}

Since $S$ is a singlet under the SM gauge group, in addition to the particle-numberconserving mass term $|S|^{2}$, it can also have the particle-number-violating mass terms $S^{2}$ and $S^{* 2}$. These mass terms induce particle-antiparticle oscillations $S \leftrightarrow S^{*}$, which are problematic as they erase the asymmetry in the dark matter sector. To avoid this problem, the oscillation rate has to be small, namely,

$$
\Gamma_{\mathrm{osc}}=\frac{\Delta m}{2} \lesssim \frac{\mathcal{C}}{M_{P}}\left(\frac{m_{\mathrm{DM}}}{25}\right)^{2}
$$


where $\Delta m$ denotes the mass splitting between the dark matter particle and its antiparticle induced by the particle-number-violating mass terms, and the right-hand side is the Hubble parameter when the dark matter decouples from the thermal bath. In the presence of the mass terms $\mu^{2}\left(S^{2}+S^{* 2}\right) / 2$, the mass splitting is given by $\Delta m=\mu^{2} / m_{\mathrm{DM}}$, and thus Eq. (52) leads to

$$
\mu \lesssim \frac{m_{\mathrm{DM}}}{25}\left(\frac{2 \mathcal{C} m_{\mathrm{DM}}}{M_{P}}\right)^{\frac{1}{2}} \simeq 2 \times 10^{-6} \times\left(\frac{m_{\mathrm{DM}}}{1 \mathrm{TeV}}\right)^{\frac{3}{2}} \mathrm{GeV}
$$

In $\mathrm{SO}(10)$, both $\mathbf{1 6}^{2}$ and $\mathbf{1 4 4 ^ { 2 }}$ are forbidden by the gauge symmetry, and thus the particle-number-violating mass terms are absent. The intermediate gauge symmetries also forbid such mass terms. Below the intermediate gauge scale, however, the VEV of the $\mathbf{1 2 6}$ Higgs field can generate the particle-number-violating mass terms via the interactions

$$
\mathcal{L}_{\text {int }}=-\kappa_{2} R_{\mathrm{DM}} R_{\mathrm{DM}} R_{2}^{*}-\lambda_{12}^{126}\left(R_{\mathrm{DM}} R_{\mathrm{DM}}\right)\left(R_{1} R_{2}^{*}\right) \overline{126}+\text { h.c. }
$$

where $R_{\mathrm{DM}}=\mathbf{1 6}$ or $\mathbf{1 4 4}$ denotes the dark matter multiplet, $R_{1}$ is the GUT Higgs field, $R_{2}=\mathbf{1 2 6}$ is the intermediate-scale Higgs field, and the subscripts after the parentheses denote the $\mathrm{SO}(10)$ representation formed by the product in them. To satisfy the bound (53), we need to suppress the couplings $\kappa_{2}$ and $\lambda_{12}^{126}$. Once they are taken to be small, they remain small under the renormalization flow. By making these couplings small, we can also suppress the particle-number-violating couplings with the Higgs boson, such as $S^{2}|H|^{2}$, which are induced by the exchange of the $\mathbf{1 2 6}$ Higgs and lead to the particlenumber-violating mass terms after electroweak symmetry breaking.

\subsection{Thermal transfer}

If the asymmetry in the dark sector is transferred from the $B-L$ asymmetry in the SM sector through effective interactions (11), the dark matter mass is uniquely determined by the thermal relation (36). The lowest-dimension effective operator which has the form of $(11)$ is

$$
\mathcal{L}_{\text {eff }}^{(7)}=\frac{c_{7}}{\Lambda^{3}} S^{2} H^{2} \overline{L^{c}} L+\text { h.c. , }
$$

which can be induced by the exchange of the intermediate-scale particles. We thus take $\Lambda=M_{\text {int }}$ with other possible suppression factors included in the coefficient $c_{7}$. $T_{\text {eq }}$ for this operator is then given by Eq. (14):

$$
T_{\text {eq }}=M_{\text {int }}\left[\frac{8 \pi^{3} \mathcal{C} M_{\mathrm{int}}}{c_{7}^{2} M_{P}}\right]^{\frac{1}{5}} .
$$


If $T_{\text {eq }} \gg m_{\mathrm{DM}}$, this interaction decouples from the thermal bath much before the decoupling of the dark matter, and in particular we can set $k\left(z_{\mathrm{dec}}\right)=1$ in Eq. (36). By setting $n_{\mathrm{DM}}=1, n_{H}=1, Y_{\mathrm{DM}}=0$, and $X_{\mathrm{DM}}=Q_{B-L}^{\mathrm{DM}}=1$, we then obtain

$$
m_{\mathrm{DM}}=m_{N} \frac{79}{22}\left(\frac{\Omega_{c} h^{2}}{\Omega_{B} h^{2}}\right)\left[\frac{B_{\mathrm{SM}}}{(B-L)_{\mathrm{SM}}}\right] \simeq 6.0 \mathrm{GeV},
$$

where we have used Eq. (35). However, such a small dark matter mass has already been excluded by the constraint on the Higgs invisible decay width as shown in Fig. 1 .

If $T_{\text {eq }} \lesssim m_{\mathrm{DM}}$, on the other hand, the dark matter mass given by Eq. (36) can be increased due to the Boltzmann factor $k\left(z_{\mathrm{dec}}\right)$. In terms of the intermediate scale $M_{\mathrm{int}}$, the inequality $T_{\text {eq }} \lesssim m_{\text {DM }}$ reads

$$
M_{\mathrm{int}} \lesssim m_{\mathrm{DM}}^{\frac{5}{6}}\left[\frac{c_{7}^{2} M_{P}}{8 \pi^{3} \mathcal{C}}\right]^{\frac{1}{6}} \simeq c_{7}^{\frac{1}{3}} \times\left(\frac{m_{\mathrm{DM}}}{1 \mathrm{TeV}}\right)^{\frac{5}{6}} \times 10^{5} \mathrm{GeV}
$$

As discussed in Sec. 3.1, however, there is no candidate in a model with minimal field content which predicts such a low intermediate scale.9 We therefore conclude that the thermal transfer scenario does not work for the scalar singlet asymmetric dark matter candidate in $\mathrm{SO}(10)$.

\subsection{Cogenesis}

Next, we discuss the cogenesis scenario. In this case, the asymmetry in the dark matter sector is directly produced via the decay of intermediate-scale particles and does not communicate to the SM sector after the generation of the asymmetry. To that end, for instance, we may utilize the decay of right-handed neutrinos by introducing a new singlet fermion $\psi_{S}$ around the intermediate scale. The singlet fermion can have a coupling with the scalar singlet dark matter and right-handed neutrinos if the singlet fermion is in a $\mathbf{1}, \mathbf{4 5}$, or $\mathbf{2 1 0}$ of $\mathrm{SO}(10){ }^{10}$ For brevity, we take it to be a $\mathbf{1}$ in the following discussion. Such a singlet field does not affect the running of gauge couplings, and thus does not spoil gauge coupling unification. If the mass of the new singlet fermion is smaller than the masses of right-handed neutrinos, the right-handed neutrinos can decay into the singlet fermion and the scalar singlet dark matter. If the new couplings contain CP phases, the dark matter asymmetry is produced in the decay process just like leptogenesis. The

\footnotetext{
${ }^{9}$ We note that one can construct a non-minimal model with a low intermediate scale. This can be done for example, if the intermediate gauge group is broken in two steps to the SM. While one of the intermediate scales remains relatively large, the second may be as low as $\sim 1 \mathrm{TeV}[26]$.

${ }^{10}$ If $R_{\mathrm{DM}}=\mathbf{1 4 4}$, a 54 is also possible.
} 
singlet fermion can decay into the dark matter and SM particles via the exchange of right-handed neutrinos afterward. Although this decay process modifies the primordial asymmetry in the dark sector, it does not completely erase the asymmetry in general since the asymmetry generated at the late time depends on a different combination of the Yukawa couplings from that for the primordial asymmetry.

Alternatively if the mass of $\psi_{S}$ is greater than $m_{\nu_{R}}$, then if $\psi_{S}$ is produced during reheating, its out-of-equilibrium decays will produce an asymmetry in the dark matter scalar $S$. The out-of-equilibrium decay of $\nu_{R}$ produces a lepton asymmetry as in ordinary leptogenesis. In either case, the net dark matter asymmetry depends on the branching fractions of right-handed neutrinos and the new CP phase, but generically it is the same order as the $B-L$ asymmetry in the SM sector, which may explain why $\Omega_{c} h^{2}$ and $\Omega_{B} h^{2}$ are the same order of magnitude.

Though the cogenesis scenario may work in this case, as shown in Fig. 1, the allowed dark matter mass region is stringently restricted and can be soon tested in future experiments (except for the very narrow region around $m_{\mathrm{DM}} \simeq m_{h} / 2$ ). This motivates us to move to the next-to-minimal models, which will be discussed in the subsequent section.

\section{Next-to-minimal models}

In the models we considered in the previous sections, the dark matter particle develops an asymmetric part in its density, either through transfer from an asymmetry of the SM particles or by cogenesis, and preserves it as (a part of) the dark matter relic observed today. As we have seen, these models are severely constrained leaving only the scalar singlet dark matter model of cogenesis with its mass limited to a narrow window around $m_{h} / 2$, (the window around $\sim 1 \mathrm{TeV}$ is also possible, though in this case most of the dark matter abundance originates from the ordinary thermal relic). However, we may find additional models if we relax the notion of the asymmetric dark matter - namely, the constraints discussed above can be relieved if the dark matter relic abundance is only required to have an asymmetric origin while it can be totally symmetric today. We discuss this possibility in this section.

More specifically, we consider dark matter models that achieve the relic density in two steps, similar to models considered in Refs. 14, 16. In these models, two $\mathbb{Z}_{2}$-odd particles (or multiplets) $X_{1}$ and $X_{2}$ are introduced near the TeV scale. $X_{1}$ is the lighter one whose relic density eventually originates from the $B-L$ asymmetry in two steps: i) $X_{2}$ obtains asymmetric density either by cogenesis or by asymmetry transfer from SM particles, and 
then ii) the asymmetric density in $X_{2}$ is converted to the relic density of $X_{1}$ through $X_{2}$ decay. In order to annihilate the symmetric part of the thermal abundance efficiently, $X_{1}$ needs to have sizable couplings with the SM sector. To that end, we assume that $X_{1}$ has a charge under the $\mathrm{SU}(2)_{L} \otimes \mathrm{U}(1)_{Y}$ gauge interactions. In the models we present below, $X_{1}$ is a Majorana fermion. $X_{2}$ needs to have a long enough lifetime to decay after depletion of $X_{1}$ symmetric density; otherwise the determination of $X_{1}$ relic density is similar to that in the usual thermal relic scenario.

In the rest of this section we will present two non-minimal models where the asymmetry of $X_{2}$ is acquired by cogenesis and by transfer, respectively. In Sec. 4.1, we extend the cogenesis model discussed in Sec. 3.5 so that the scalar singlet plays the role of $X_{2}$ and the dark matter particle $X_{1}$ is a singlet-doublet Majorana particle. In Sec. 4.2, we consider a model where $X_{2}$ is a stop-like particle whose asymmetry is transferred from the top quark by a Yukawa interaction.

\subsection{Extension of cogenesis model}

In the model considered in Sec. 3.5, the asymmetry in the dark matter sector can be generated by the lightest right-handed neutrino decay $N_{1} \rightarrow S+\psi_{S}$. In addition, we introduce an additional $\mathbb{Z}_{2}$-odd Dirac doublet $\psi_{D}$ around the TeV scale. The neutral component of the doublet $\psi_{D}^{0}$ is mixed with the fermionic singlet $\psi_{S}$ through the Yukawa coupling

$$
\mathcal{L}_{\text {Yukawa }}=-\kappa \psi_{S} \psi_{D} H+\text { h.c. }
$$

after the Higgs field develops a VEV. Furthermore, we choose the masses of $\psi_{S}, \psi_{D}$, and $S-m_{\psi_{S}}, m_{\psi_{D}}$, and $m_{S}$, respectively - such that $m_{\psi_{S}}, m_{\psi_{D}}<m_{S}<M_{1}$. The lightest $\mathbb{Z}_{2}$-odd particle is a $\psi_{D}^{0}-\psi_{S}$ mixture which we denote by $\chi$ in the following text becomes the dark matter candidate.

There are two ways for $\chi$ to achieve the correct dark matter relic density. The first possibility is that $S$ decays early into $\chi$, and then $\chi$ annihilates through its $\psi_{D}^{0}$ component by a gauge interaction. In this case, the primordial asymmetry generated in the dark sector is washed out since $\chi$ is a Majorana particle, and thus this case is just the ordinary thermal relic scenario. Here, we consider the other possibility; both $S$ and $\chi$ nearly fully annihilate their symmetric density before the asymmetric part of $S$ decays into $\chi$. In this case, the annihilation cross sections of both $S$ and $\chi$ are larger than those required in the thermal relic scenario so that the symmetric part of their abundance is suppressed after the annihilations freeze out. 
By requiring a low symmetric relic density, we can constrain the masses of $S$ and $\chi$. The relic abundance of an $\mathrm{SU}(2)_{L}$ doublet Dirac dark matter candidate is saturated by the symmetric part if its mass is about $1 \mathrm{TeV}[54]$. Thus, if we require that the density of asymmetric origin makes up over $90 \%$ of the total relic density, we can set a bound on the dark matter particle mass $m_{\chi} \lesssim 1 \mathrm{TeV} / \sqrt{10} \sim 350 \mathrm{GeV}$. The DM-nucleon scattering cross section for almost pure $\mathrm{SU}(2)_{L}$ doublet dark matter is found to be very small $\left(\sigma_{\mathrm{SI}} \lesssim 10^{-49} \mathrm{~cm}^{2}\right)$ 70 and thus this candidate can evade the direct detection limits.11 We recall from Fig. 1 that $m_{S} \lesssim 700 \mathrm{GeV}$ if its symmetric density contributes less than $10 \%$ of the dark matter relic density and if we assume perturbativity of $\lambda_{S H}$.

The decay of $S$ proceeds via its coupling to the matter $\mathbf{1 6}$ and the singlet in $\chi$ so that $S$ to $\chi$ is mediated by right-handed neutrinos: $S \rightarrow \chi+N_{i}^{(*)} \rightarrow \chi+L_{i}+H$, where $N_{i}^{(*)}$ represent the virtual intermediate $N_{i}$ and $L_{i}$ are the left-handed lepton doublets. The decay width of $S$ is estimated as

$$
\Gamma_{S} \simeq \sum_{i} \frac{\lambda_{i}^{2} y_{i}^{2}}{3 \times 2^{8} \pi^{3}} \frac{m_{S}^{3}}{M_{i}^{2}},
$$

where $\lambda_{i}$ denote the $\chi^{-S}-N_{i}$ couplings and $y_{i}=y_{i i}$. If $m_{S} \simeq m_{\chi}$, then this decay width is further suppressed by a phase space factor. Now suppose that the exchange of the lightest right-handed neutrino $N_{1}$ dominates the scattering amplitude. Here, we note that the relevant couplings $\lambda_{1}$ and $y_{1}$ are restricted by the out-of-equilibrium decay condition (5): $\left(\lambda_{1}^{2}+y_{1}^{2}\right) \lesssim 8 \pi \mathcal{C} M_{1} / M_{P}$. This then gives

$$
\Gamma_{S} \lesssim \frac{1}{3 \times 2^{8} \pi^{3}} \frac{m_{S}^{3}}{M_{1}^{2}} \times \frac{4^{2} \pi^{2} \mathcal{C}^{2} M_{1}^{2}}{M_{P}^{2}}=\frac{\mathcal{C}^{2} m_{S}^{3}}{48 \pi M_{P}^{2}},
$$

and thus a lower limit on the lifetime of $S, \tau_{S}$, is obtained as

$$
\tau_{S} \gtrsim 4 \times 10^{5} \times\left(\frac{500 \mathrm{GeV}}{m_{S}}\right)^{3} \mathrm{~s} .
$$

Such slow decay can re-process the light element abundances produced by the Big-Bang nucleosynthesis (BBN), and thus is strongly constraint by the success of BBN calculation. It is shown in Ref. [76 that the BBN constraint starts to come into play when $\tau_{S} \gtrsim 100 \mathrm{~s}$.

\footnotetext{
${ }^{11}$ If $\chi$ is a well-mixed state of singlet and doublet components, the dark matter-nucleon scattering is induced by the Higgs boson exchange process, which is severely constrained by the direct detection experiments 71,72. However, there is a specific parameter region, so-called blind spot $[7375]$, where the direct detection bound is evaded even though the singlet-doublet mixing is sizable. In this region, the symmetric part of dark matter relic agrees with the observed dark matter density even if the dark matter mass is as large as $\sim 1.5 \mathrm{TeV}[72$; therefore, for the symmetric origin of the dark matter abundance to be less than $10 \%, m_{\mathrm{DM}} \lesssim 1.5 \mathrm{TeV} / \sqrt{10} \sim 500 \mathrm{GeV}$ is required in the case of the blind spot.
} 
To make $\tau_{S}<100 \mathrm{~s}$, the decay of $S$ should be dominated by the exchange of heavier righthanded neutrinos, since the Yukawa couplings for these heavier right-handed neutrinos, $y_{2}$ and $y_{3}$, are in general not limited by the condition (5) since there is no reason to impose that $N_{2,3}$ decay out-of-equilibrium. Then the decay of $S$ can be dominated by $N_{2,3}$ by assuming $\lambda_{2,3} \gg \lambda_{1}$. We however note that it is necessary to ensure that the asymmetry wash-out scatterings $L L \leftrightarrow H^{*} H^{*}$ and $S S \leftrightarrow \psi_{S} \psi_{S}$ decouple at the temperature $T_{B L}$ defined in Sec. 2.2. Suppose the exchange of $N_{i}, i \neq 1$ dominates the $S$ decay. Then, let us estimate the bound on $y_{i}$ and $\lambda_{i}$ coming from the decoupling conditions of the wash-out processes; this is given by Eq. (40) as $y_{i}^{2}, \lambda_{i}^{2} \lesssim M_{i} /\left(0.017 \sqrt{T_{B L} M_{P}}\right)$. The decay rate of $S$ is then

$$
\Gamma_{S} \lesssim \frac{1}{3 \times 2^{8} \pi^{3}(0.017)^{2}} \frac{m_{S}^{3}}{M_{P} T_{B L}}
$$

and thus

$$
\tau_{S} \gtrsim 4 \times 10^{-3} \times\left(\frac{500 \mathrm{GeV}}{m_{S}}\right)^{3}\left(\frac{T_{B L}}{10^{10} \mathrm{GeV}}\right) \mathrm{s} .
$$

Therefore, the lifetime of $S$ can be short enough to evade the BBN bound. This result is qualitatively straightforward: a larger $S$ decay rate requires larger couplings $y_{i}$ and $\lambda_{i}$, which cause later decoupling of the wash-out processes. The $B-L$ generation should then occur at a later time, which requires a low $T_{B L}$.

Finally we fit these particles into an $\mathrm{SO}(10)$ unification model with an intermediate gauge group as described in Sec. 2.1. In this specific model, the $\mathrm{SO}(10)$ gauge symmetry is broken by the VEV of a singlet in $\mathbf{2 1 0}_{R}$ into the intermediate symmetry group $G_{\text {int }}=$ $\mathrm{SU}(4)_{C} \otimes \mathrm{SU}(2)_{L} \otimes \mathrm{SU}(2)_{R}$ at $M_{\mathrm{GUT}}$. $G_{\text {int }}$ is then broken down to the SM gauge group $G_{\mathrm{SM}}$ at $M_{\text {int }}$ by the VEV of the $(\mathbf{1 0}, \mathbf{1}, \mathbf{3})_{C}$ component in a $\mathbf{1 2 6}_{C}$ and the $(\mathbf{1 5}, \mathbf{1}, \mathbf{1})_{R}$ component in the $\mathbf{2 1 0}_{R}$. The subscript $R$ and $C$ stand for real and complex fields, respectively. The numbers in the parenthesis are the $G_{\text {int }}$ quantum numbers of the fields. The particle content except the SM fermions and right-handed neutrinos are summarized in Table 2 . There, the first column shows the particle content around the electroweak or TeV scale. The second and third columns show the quantum numbers under $G_{\text {int }}$ and the $\mathrm{SO}(10)$ representation, respectively. $W$ stands for a Weyl field. Most of the particles in the representation shown in the second column have intermediate-scale masses, except for the components listed in the first column. Other components of the $\mathrm{SO}(10)$ representation are assumed to lie around $M_{\mathrm{GUT}}$. The dark sector particles $\psi_{S}, \psi_{D}$ and $S$ belong to Weyl 1, Weyl 10 and complex scalar 16 representations, respectively. The SM Higgs doublet, which breaks $G_{\mathrm{SM}}$, is a mixture of doublets in $(\mathbf{1}, \mathbf{2}, \mathbf{2})_{C}$ of $\mathbf{1 0}_{C}$ and $(\mathbf{1 0}, \mathbf{2}, \mathbf{2})_{C}$ of $\mathbf{2 1 0}_{C}$. 
The latter component cannot couple to the SM fermions or right-handed neutrinos but it can couple to the components of the $\mathbf{1 6}_{S}$. At the intermediate scale $(\mathbf{1 0 , 2}, \mathbf{2})_{C}$ and $(\mathbf{1 5}, \mathbf{1}, \mathbf{1})_{R}$ are required only for achieving unification. With this particle content, the one loop result of the scales and unification coupling are

$$
M_{\mathrm{int}}=10^{11.8} \mathrm{GeV}, \quad M_{\mathrm{GUT}}=10^{15.7} \mathrm{GeV}, \quad \alpha_{\mathrm{GUT}}=0.027
$$

The high intermediate scale guarantees small neutrino masses by the type-I seesaw mechanism. The constraint from proton decay is evaded by the high unification scale.

Table 2: Particle content of the extended cogenesis model. The first column shows the particle content around the electroweak or TeV scale. The second column and the third column show the quantum number under $G_{\text {int }}$ and the $S O(10)$ representation, respectively.

\begin{tabular}{ccc}
\hline \hline $\mathrm{EW}$ & $\mathrm{SU}(4)_{C} \otimes \mathrm{SU}(2)_{L} \otimes \mathrm{SU}(2)_{R}$ & $\mathrm{SO}(10)$ \\
\hline$S$ & $(\mathbf{4}, \mathbf{1}, \mathbf{2})_{C}$ & $\mathbf{1 6}_{C}$ \\
$\psi_{D}$ & $(\mathbf{1}, \mathbf{2}, \mathbf{2})_{W}$ & $\mathbf{1 0}_{W}$ \\
$\psi_{S}$ & $(\mathbf{1}, \mathbf{1}, \mathbf{1})_{W}$ & $\mathbf{1}_{W}$ \\
$H$ & $(\mathbf{1 0}, \mathbf{2}, \mathbf{2})_{C}$ & $\mathbf{2 1 0}_{R}$ \\
$H$ & $(\mathbf{1}, \mathbf{2}, \mathbf{2})_{C}$ & $\mathbf{1 0}_{C}$ \\
& $(\mathbf{1 5}, \mathbf{1}, \mathbf{1})_{R}$ & $\mathbf{2 1 0}_{R}$ \\
& $(\mathbf{1 0}, \mathbf{1}, \mathbf{3})_{R}$ & $\mathbf{1 2 6}_{C}$ \\
\hline \hline
\end{tabular}

\subsection{Asymmetry transfer by Yukawa coupling}

In this section we present another possibility where the asymmetry in $X_{2}$ is obtained from a Yukawa coupling of the form $X_{2} \bar{X}_{1} f$ with $f$ representing the SM fermions. $X_{1}$ and $X_{2}$ are taken to be a Majorana fermion and a complex scalar multiplet, respectively. The chemical potentials of $X_{1}$ and $X_{2}$ are determined by the neutrality of the Majorana particle $X_{1}$ and by this Yukawa interaction, respectively:

$$
\mu_{X_{1}}=0, \quad \mu_{X_{2}}=-\mu_{f}
$$

Similarly to the extended cogenesis model, $X_{2}$ is supposed to decay into $X_{1}$ after the $X_{1}-X_{1}$ and $X_{2}-X_{2}^{*}$ annihilation processes decouple. If these annihilation processes deplete 
$X_{1}$ and the symmetric part of $X_{2}$ density efficiently, the relic abundance will be determined by the asymmetric part of the $X_{2}$ density before its decay. As we will see, the slow decay $X_{2} \rightarrow X_{1}+\bar{f}$ requires a small mass gap between $X_{2}$ and $X_{1}$. At low temperature $T<m_{X_{1}}$, the asymmetry is transferred through the scattering $f+X_{1,2} \rightarrow A+X_{2,1}$ with SM fermions propagating in the $t$-channel and $A$ is any light gauge boson which couples to $f$. The decoupling temperature of the asymmetric transfer $T_{\operatorname{dec}}$ is thus determined by the decoupling of this $t$-channel scattering process.

As a concrete example of this model, we choose $X_{2}$ as a right-handed stop-like particle $\widetilde{t}_{R}$, which is a color triplet, weak isospin singlet and has hypercharge $2 / 3 . X_{1}$ is chosen as a mixture of a singlet Majorana fermion $\psi_{S}$ and the neutral component of a doublet $\psi_{D}^{0}$, as in the extended cogenesis model discussed in the previous subsection. Furthermore, we assume $\widetilde{t}_{R}$ only couples to the right-handed top quark $t_{R}$ through the Yukawa coupling

$$
\mathcal{L}=\lambda_{t} \bar{t}_{R} \psi_{S} \widetilde{t}_{R}+\text { h.c. }
$$

which resembles the bino-stop-top coupling in the minimal supersymmetric Standard Model.

The $\mathrm{SO}(10)$ completion of this model on top of the three generations of the SM $\mathbf{1 6}$ is summarized in Table 3 . The $\mathrm{SO}(10)$ symmetry is broken by a $\mathbf{2 1 0}_{R}$ to $G_{\text {int }}=$ $\mathrm{SU}(4)_{C} \otimes \mathrm{SU}(2)_{L} \otimes \mathrm{SU}(2)_{R}$, which is broken subsequently to $G_{\mathrm{SM}}$ by the VEV of $(\mathbf{1 0}, \mathbf{1}, \mathbf{3})_{C}$ in a $\mathbf{1 2 6}_{C} \cdot \psi_{S}, \psi_{D}$ and $\widetilde{t}_{R}$ belong to Weyl 45, Weyl 10, and complex scalar 16 representations, respectively ${ }^{12}$ The Yukawa interaction 67 comes from the coupling $\mathbf{1 6}^{*} \mathbf{1 6}_{f} \mathbf{4 5}_{W}$ where $\mathbf{1 6}_{f}$ is the multiplet composed of the third generation SM fermions and righthanded neutrino. $G_{\mathrm{SM}}$ is broken by the VEV of the following doublets: $(\mathbf{1}, \mathbf{2}, \mathbf{2})_{R}$ of $\mathbf{1 0}_{R}$, $(\mathbf{1 5}, \mathbf{2}, \mathbf{2})_{C}$ of $\mathbf{1 2 6}_{C}$, and $(\mathbf{1 0}, \mathbf{2}, \mathbf{2})_{C}$ of $\mathbf{2 1 0}_{R}$. The SM Higgs doublet is a mixture of the above doublets. The latter two multiplets at the intermediate scale are necessary for achieving a sufficiently high unification scale. With this particle content, the one-loop result for the scales and unification coupling are

$$
M_{\mathrm{int}}=10^{11.3} \mathrm{GeV}, \quad M_{\mathrm{GUT}}=10^{15.7} \mathrm{GeV}, \quad \alpha_{\mathrm{GUT}}=0.035
$$

Now we consider the constraint placed on the coupling strength $\lambda_{t}$ and the particle masses. The constraint on the mass of $\chi$ from efficient annihilation is the same as that discussed in Sec. 4.1$]^{13}$ The relationship between the relic density and the dark matter

\footnotetext{
${ }^{12}$ We are required here to consider a higher representation for $\psi_{S}$ to achieve gauge coupling unification with a sufficiently high GUT scale.

${ }^{13} \mathrm{As}$ we see below, the coupling $\lambda_{t}$ is required to be very small, and thus the contribution of the
} 
Table 3: Particle content of the stop mediated asymmetry transfer model. The first column shows the particle content around the electroweak or TeV scale. The second column and the third column show the quantum number under $G_{\text {int }}$ and the $S O(10)$ representation respectively.

\begin{tabular}{ccc}
\hline \hline $\mathrm{EW}$ & $\mathrm{SU}(4)_{C} \otimes \mathrm{SU}(2)_{L} \otimes \mathrm{SU}(2)_{R}$ & $\mathrm{SO}(10)$ \\
\hline$\widetilde{t}_{R}$ & $(\mathbf{4}, \mathbf{1}, \mathbf{2})_{C}$ & $\mathbf{1 6}_{C}$ \\
$\psi_{D}$ & $(\mathbf{1}, \mathbf{2}, \mathbf{2})_{W}$ & $\mathbf{1 0}_{W}$ \\
$\psi_{S}$ & $(\mathbf{1}, \mathbf{1}, \mathbf{3})_{W}$ & $\mathbf{4 5}_{W}$ \\
$H$ & $(\mathbf{1 5}, \mathbf{2}, \mathbf{2})_{C}$ & $\mathbf{1 2 6}_{C}$ \\
$H$ & $(\mathbf{1 0}, \mathbf{2}, \mathbf{2})_{C}$ & $\mathbf{2 1 0}_{R}$ \\
$H$ & $(\mathbf{1}, \mathbf{2}, \mathbf{2})_{C}$ & $\mathbf{1 0}_{C}$ \\
& $(\mathbf{1 0}, \mathbf{1}, \mathbf{3})_{C}$ & $\mathbf{1 2 6}_{C}$ \\
\hline \hline
\end{tabular}

mass is again given by Eq. (36), with the relevant quantities for dark matter in Eq. (36) replaced with the corresponding quantities for $\widetilde{t}_{R}$; namely, we set $n_{H}=1, n_{\mathrm{DM}}=1$, $Y_{\mathrm{DM}}=2 / 3, X_{\mathrm{DM}}=1 / 3$, and replace $k\left(z_{\mathrm{dec}}\right)$ with $3 k\left(z_{\mathrm{dec}}\right)$ to take the color factor for $\widetilde{t}_{R}$ into account 14 We then have

$$
m_{\chi} \simeq m_{N}\left(\frac{\Omega_{c}}{\Omega_{B}}\right) \frac{474+144 k\left(z_{\mathrm{dec}}\right)}{185 k\left(z_{\mathrm{dec}}\right)}
$$

where $z_{\mathrm{dec}}=m_{\tilde{t}_{R}} / T_{\mathrm{dec}}$ with $T_{\mathrm{dec}}$ the decoupling temperature of the Yukawa interaction, and we have used Eq. (35). The required value for $z_{\text {dec }}$ is then obtained from the observed dark matter density using this relation, as shown in Fig. 2. We find that it is in the range of 4-6.5 for $m_{\chi}$ of $200 \mathrm{GeV}-1 \mathrm{TeV}$.

According to Fig. 2, around the decoupling temperature of the Yukawa interaction (67), the temperature is as low as $\sim 100 \mathrm{GeV}$ and thus even the dominant $t$-channel scattering process $t+\chi \leftrightarrow g+\widetilde{t}_{R}$, with $g$ a gluon, is exponentially suppressed. The reaction rate is estimated as

$$
\Gamma\left(t \chi \leftrightarrow g \tilde{t}_{R}\right) \simeq \frac{g_{3}^{2} \lambda_{t}^{2}}{\pi m_{\chi} m_{t}} \cdot\left(\frac{m_{t} T}{2 \pi}\right)^{3 / 2} e^{-m_{t} / T} .
$$

interaction (67) to the annihilation of the dark matter particles is negligible. Moreover, since the conversion process $t \chi \leftrightarrow g \widetilde{t}_{R}$ decouples before the decoupling of the dark matter (see the discussion below), coannihilation with $\widetilde{t}_{R}$ is ineffective.

${ }^{14}$ Strictly speaking, we may not directly apply Eq. $(36)$ to the present case as $\widetilde{t}_{R}$ can be in thermal bath until the time of the sphaleron decoupling, though this effect does not affect our discussion significantly. 


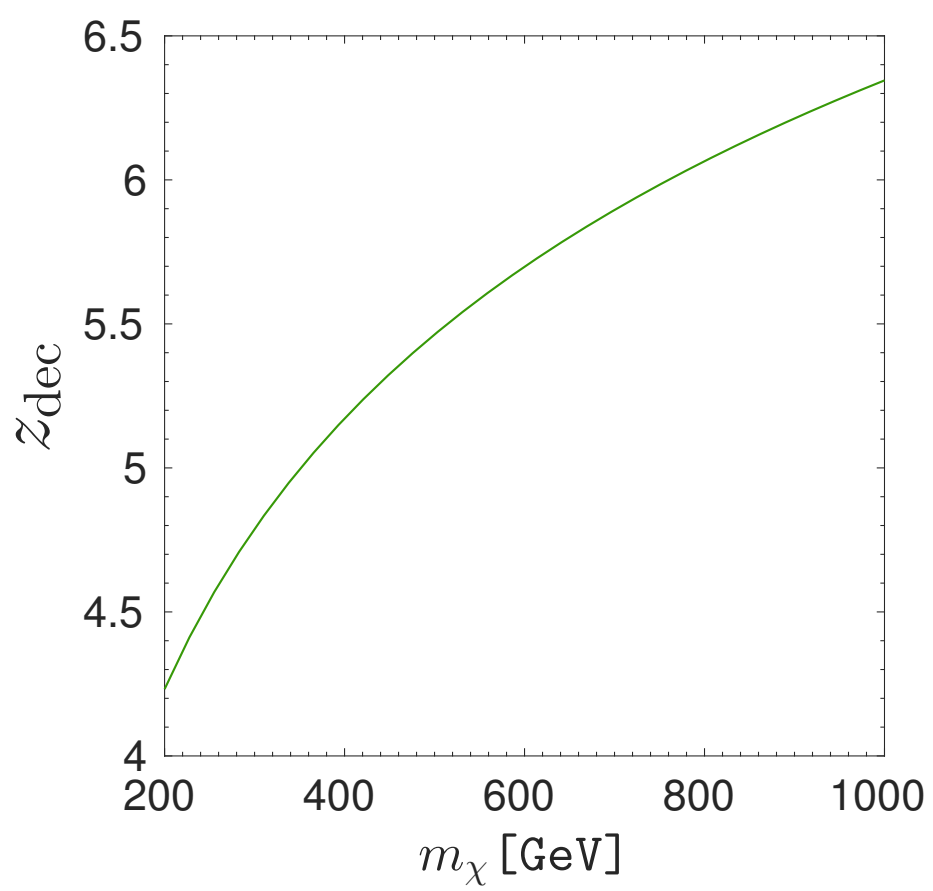

Figure 2: $z_{\mathrm{dec}}=m_{\tilde{t}_{R}} / T_{\mathrm{dec}}$ as a function of $m_{\chi}$, determined by dark matter relic density.

Through this process, any asymmetry in tops (baryon asymmetry) is transferred to an asymmetry in the $\widetilde{t}_{R}$ which subsequently decay to $\chi$. The decoupling temperature is estimated from $\Gamma\left(t \chi \leftrightarrow g \widetilde{t}_{R}\right) \simeq H$ and using the result in Fig. 2, we then obtain $\lambda_{t} \simeq$ $1.4(1.1) \times 10^{-6}$ for $m_{\chi}=200(1000) \mathrm{GeV}$.

Now let us consider the condition that $\widetilde{t}_{R}$ has a lifetime long enough to decay after the annihilation of the symmetric part of $\chi$ is over. To ensure such slow decay, we need to set $\Delta m \equiv m_{\widetilde{t}_{R}}-m_{\chi}<m_{t}$ so that the two-body decay channel $\widetilde{t}_{R} \rightarrow t \chi$ is kinematically forbidden 15 The dominant decay channel is then the three-body decay $\widetilde{t}_{R} \rightarrow b W \chi_{i}$, $i=1,2,3$ represents three mass eigenstates of $\psi_{D}^{0}-\psi_{S}$ mixing, and for simplicity we assume $\widetilde{t}_{R}$ can decay to all of them, so that the decay rate is not suppressed by the mixing angle. The decay occurs after $\chi-\chi$ annihilation if $\Gamma_{\tilde{t}_{R}}<\left.H\right|_{T_{f}}$, where $m_{\chi} / T_{f} \sim 20$ is the decoupling temperature of the annihilation. Numerical calculation of the decay rate gives a bound of $\Delta m \lesssim 110$ (160) GeV for $m_{X_{1}}=200$ (1000) GeV, assuming the three $\chi_{i}$ are degenerate in mass.

Since $\widetilde{t}_{R}$ has a relatively long lifetime $\sqrt[16]{16}$ is hadronized before it decays when pro-

\footnotetext{
${ }^{15}$ Such a small mass difference also allows $\widetilde{t}_{R}$ to evade the strong limits from stop searches at the LHC [77, 78].

${ }^{16}$ The lifetime for the mass ranges considered is $\mathcal{O}(1)$ ns, and so is clearly not a problem for BBN.
} 
duced at colliders. Such a massive charged particle (e.g., $R$-hadron) yields characteristic signatures in the detectors. The ATLAS [79] and CMS [80] collaborations have searched for heavy charged stable particles based on observables related to large ionization energy losses. Both searches require the $R$-hadron to get to the calorimeter in order to pass data selection, and this corresponds to a lifetime $\sim 4 \mathrm{~ns}$. The constraint for charged stable $R$-hadrons sets a lower bound on stable stop and sbottom masses up to $\sim 900 \mathrm{GeV}$. This implies an upper limit to the $\widetilde{t}_{R}$ lifetime, $\tau_{\widetilde{t}_{R}} \lesssim 4 \mathrm{~ns}$, otherwise the density originating from an asymmetry can only occupy up to $20 \%$ of the relic density when $m_{\tilde{t}_{R}} \sim 900 \mathrm{GeV}$. A more severe limit on $\widetilde{t}_{R}$ may in principle be obtained from the displaced vertex searches [81. However, we cannot directly apply the results in Ref. 81] to the present case due to the small mass difference $\Delta m$. Although the reconstruction efficiency of displaced vertices remains sizable even if $\Delta m \lesssim 100 \mathrm{GeV}$ [82], the trigger efficiency is reduced, which results in a weaker constraint. A dedicated study of this limit is beyond the scope of the present paper.

Finally we remark that the framework of transferring the asymmetry through Yukawa interactions can also be applied straightforwardly to other choices of $\mathbb{Z}_{2}$-odd particles. For example, we can also choose $X_{1}$ as a single Majorana triplet (thus avoiding the need for mixing among two multiplets) and $X_{2}$ as a slepton-like doublet. The asymmetry is transferred to $X_{2}$ from the lepton doublet. The decoupling temperature of asymmetry transfer in this case is however exponentially sensitive to the Yukawa coupling, since the asymmetry transfer scattering $X_{1}+\ell^{-} \leftrightarrow \gamma+X_{2}$ is mediated by a lepton and the reaction rate is dependent on $\log \left(T / m_{\ell}\right) / T$ when $m_{\ell} \ll T \ll X_{1}$. We will not discuss this model in more detail here.

\section{Conclusion}

The baryon and relic dark matter densities are known to be quite similar. It is not known, however, whether or not the origin of these densities are related.

The standard thermal mechanism for obtaining the correct dark matter relic abundance is quite robust. The annihilation of dark matter candidates with weak-scale (or $\mathrm{TeV}$ ) masses with weak-scale (or slightly weaker) interactions leaves behind a density close to that determined observationally. Of course there are many non-thermal mechanisms which may also produce the correct relic density. The calculation of the baryon density on the other hand requires a model of baryogenesis and necessarily relies on unknown quantities such as $\mathrm{C}$ and $\mathrm{CP}$ violating phases. Among the many mechanisms for generating 
the baryon asymmetry, one of the most attractive (and simplest) is the out-of-equilibrium decay of a heavy right-handed neutrino as in the original leptogenesis scenario [32]. While this scenario also relies on the values of unknown phases, very little is needed beyond the existence of the heavy right-handed states already included in $\mathrm{SO}(10)$ models and responsible for the observed low-mass neutrinos. If the generation of the baryon (or lepton) asymmetry is accompanied by the simultaneous generation of an asymmetry in dark matter [10] (assuming then that the symmetric component is driven to a low value through thermal annihilation), we can understand why these two numbers are close.

In this paper, we have attempted to construct a model of asymmetric $\mathrm{SO}(10)$ dark matter. In addition to being able to account for neutrino masses and leptogenesis quite naturally, $\mathrm{SO}(10)$ models which break through an intermediate scale gauge group contain an unbroken $\mathbb{Z}_{2}$ symmetry which can account for the stability of dark matter. This is similar to $R$-parity in supersymmetric models, but appears directly from gauge symmetry breaking. Utilizing the presence of an intermediate scale, these $\mathrm{SO}(10)$ models may account for gauge coupling unification without supersymmetry. However despite the many possible constructions of models with different intermediate scale gauge groups and dark matter representations, there are relatively few models which allow gauge coupling unification with phenomenologically acceptable intermediate and GUT mass scales [23]. The question we posed here, is whether any model can be constructed for which the dark matter density is connected to the baryon asymmetry.

From the list of possible $\mathrm{SO}(10)$ dark matter candidates, only a subset of them can accommodate an asymmetry (for example all Majorana fermion candidates are excluded). Furthermore, the universe of candidates is further limited when constraints from direct detection experiments are applied. We must also require that the symmetric thermal component annihilate efficiently. We first considered minimal models, where the field content is limited to the SM matter representations, the Higgs representations needed to break the GUT, intermediate and SM gauge symmetries, along with a single dark matter representation. For minimal models, we argued that the only candidate for asymmetric dark matter is the complex scalar singlet residing in a $\mathbf{1 6}$ or a $\mathbf{1 4 4}$ of $\mathrm{SO}(10)$. We found that the constraints from annihilation, and direct detection precluded the ability to transfer the asymmetry from the SM sector to the dark matter sector through thermal interactions unless the intermediate scale is relatively low (see Eq. (58)). However, without further complicating the model, gauge coupling unification would be lost. In contrast, the addition of a single fermion singlet would allow the cogenesis mechanism to simultaneously generate the dark matter asymmetries along with the lepton asymmetry produced during 
leptogenesis.

We also considered two extended models in which the asymmetry in some field (labelled $X_{2}$ here) is generated from (in the case of the transfer mechanism) or with (in the case of cogenesis) the baryon and lepton asymmetry. In the cogenesis model, the asymmetry in the scalar singlet (from the 16) decays to the dark matter which is a mixed state of the $\mathrm{SO}(10)$ singlet and a weak bi-doublet from a $\mathbf{1 0}$ of $\mathrm{SO}(10)$. The intermediate and GUT scales in this model are sufficiently high to easily produce light neutrino masses through the see-saw and provide sufficiently long proton lifetimes. In the specific transfer model described, the baryon asymmetry (stored in top quarks) is transferred to a right-handed scalar color triplet (also in a 16) which decays to the dark matter which is again a mixed state of a SM singlet (though now in a $\mathbf{4 5}$ of $\mathrm{SO}(10)$ ) and the same weak bi-doublet from a 10. This model also has sufficiently high intermediate and GUT scales.

The fact that present-day experimental constraints, particularly from direct detection experiments place strong constraints on these models, there remains hope that these experiments will shed further light on the nature of dark matter and whether or not the dark matter may be ultimately related to the baryon density of the Universe.

\section{Acknowledgments}

This work was supported in part by DOE grant de-sc0011842 at the University of Minnesota.

\section{References}

[1] P. A. R. Ade et al. [Planck Collaboration], Astron. Astrophys. 594, A13 (2016) arXiv:1502.01589 [astro-ph.CO]].

[2] M. Betoule et al. [SDSS Collaboration], Astron. Astrophys. 568, A22 (2014) arXiv:1401.4064 [astro-ph.CO]].

[3] F. Beutler et al., Mon. Not. Roy. Astron. Soc. 416, 3017 (2011) arXiv:1106.3366 [astro-ph.CO]]; L. Anderson et al. [BOSS Collaboration], Mon. Not. Roy. Astron. Soc. 441, no. 1, 24 (2014) arXiv:1312.4877 [astro-ph.CO]]; A. J. Ross, L. Samushia, C. Howlett, W. J. Percival, A. Burden and M. Manera, Mon. Not. Roy. Astron. Soc. 449, no. 1, 835 (2015) arXiv:1409.3242 [astro-ph.CO]].

[4] P. Hut, Phys. Lett. B69 (1977) 85. 
[5] B. W. Lee and S. Weinberg, Phys. Rev. Lett. 39 (1977) 165.

[6] M. I. Vysotsky, A. D. Dolgov and Y. B. Zeldovich, Pisma Zh. Eksp. Teor. Fiz. 26 (1977) 200.

[7] E. W. Kolb and K. A. Olive, Phys. Rev. D33 (1986) 1202; E: 34 (1986) 2531; L. M. Krauss, Phys. Lett. 128B (1983) 37.

[8] R. Watkins, M. Srednicki and K. A. Olive, Nucl. Phys. B310 (1988) 693.

[9] K. A. Olive, Nucl. Phys. B 190, 483 (1981).

[10] P. Hut and K. A. Olive, Phys. Lett. B87 (1979) 144.

[11] S. Nussinov, Phys. Lett. B 165, 55 (1985); G. B. Gelmini, L. J. Hall and M. J. Lin, Nucl. Phys. B 281, 726 (1987); S. M. Barr, R. S. Chivukula and E. Farhi, Phys. Lett. B 241, 387 (1990); S. M. Barr, Phys. Rev. D 44, 3062 (1991); D. B. Kaplan, Phys. Rev. Lett. 68, 741 (1992); S. Dodelson, B. R. Greene and L. M. Widrow, Nucl. Phys. B 372, 467 (1992); V. A. Kuzmin, Phys. Part. Nucl. 29, 257 (1998) [Fiz. Elem. Chast. Atom. Yadra 29, 637 (1998)] [Phys. Atom. Nucl. 61, 1107 (1998)] [hepph/9701269; ; D. Hooper, J. March-Russell and S. M. West, Phys. Lett. B 605, 228 (2005) hep-ph/0410114]; R. Kitano and I. Low, Phys. Rev. D 71, 023510 (2005) hepph/0411133; R. Kitano and I. Low, hep-ph/0503112; G. R. Farrar and G. Zaharijas, Phys. Rev. Lett. 96, 041302 (2006) [hep-ph/0510079]; D. E. Kaplan, M. A. Luty and K. M. Zurek, Phys. Rev. D 79, 115016 (2009) arXiv:0901.4117 [hep-ph]]; J. MarchRussell and M. McCullough, JCAP 1203, 019 (2012) |arXiv:1106.4319 [hep-ph]]; H. Davoudiasl, D. E. Morrissey, K. Sigurdson and S. Tulin, Phys. Rev. D 84, 096008 (2011) arXiv:1106.4320 [hep-ph]]; Y. Cui, L. Randall and B. Shuve, JHEP 1108, 073 (2011) arXiv:1106.4834 [hep-ph]]; N. Okada and O. Seto, Phys. Rev. D 86, 063525 (2012) arXiv:1205.2844 [hep-ph]]; S. Davidson and M. Elmer, JHEP 1210, 148 (2012) arXiv:1208.0551 [hep-ph]]; K. Y. Choi and O. Seto, Phys. Rev. D 88, no. 3, 035005 (2013) arXiv:1305.4322 [hep-ph]]; B. Bhattacherjee, S. Matsumoto, S. Mukhopadhyay and M. M. Nojiri, JHEP 1310, 032 (2013) arXiv:1306.5878 [hepph]]; H. Davoudiasl, Phys. Rev. Lett. 114, no. 5, 051802 (2015) arXiv:1409.4823 [hep-ph]]; H. Fukuda, S. Matsumoto and S. Mukhopadhyay, Phys. Rev. D 92, no. 1, 013008 (2015) arXiv:1411.4014 [hep-ph]]; N. Fonseca, L. Necib and J. Thaler, JCAP 1602, no. 02, 052 (2016) [arXiv:1507.08295 [hep-ph]]; N. Bernal, C. S. Fong and N. Fonseca, JCAP 1609, no. 09, 005 (2016) [arXiv:1605.07188 [hep-ph]]. 
[12] M. Ibe, S. Matsumoto and T. T. Yanagida, Phys. Lett. B 708, 112 (2012) arXiv:1110.5452 [hep-ph]].

[13] K. Blum, A. Efrati, Y. Grossman, Y. Nir and A. Riotto, Phys. Rev. Lett. 109, 051302 (2012) arXiv:1201.2699 [hep-ph]].

[14] G. Servant and S. Tulin, Phys. Rev. Lett. 111, no. 15, 151601 (2013) arXiv:1304.3464 [hep-ph]].

[15] S. M. Boucenna, M. B. Krauss and E. Nardi, Phys. Lett. B 748, 191 (2015) arXiv:1503.01119 [hep-ph]].

[16] M. Dhen and T. Hambye, Phys. Rev. D 92, no. 7, 075013 (2015) arXiv:1503.03444 [hep-ph]].

[17] H. Davoudiasl and R. N. Mohapatra, New J. Phys. 14, 095011 (2012) arXiv:1203.1247 [hep-ph]]; K. Petraki and R. R. Volkas, Int. J. Mod. Phys. A 28, 1330028 (2013) arXiv:1305.4939 [hep-ph]]; K. M. Zurek, Phys. Rept. 537, 91 (2014) arXiv:1308.0338 [hep-ph]].

[18] M. Kadastik, K. Kannike and M. Raidal, Phys. Rev. D 80 (2009) 085020 [Erratumibid. D 81 (2010) 029903] arXiv:0907.1894 [hep-ph]].

[19] M. Kadastik, K. Kannike and M. Raidal, Phys. Rev. D 81, 015002 (2010) arXiv:0903.2475 [hep-ph]]; M. Frigerio and T. Hambye, Phys. Rev. D 81 (2010) 075002 arXiv:0912.1545 [hep-ph]].

[20] Y. Mambrini, K. A. Olive, J. Quevillon and B. Zaldivar, Phys. Rev. Lett. 110 (2013) 24, 241306 [arXiv:1302.4438 [hep-ph]].

[21] Y. Mambrini, N. Nagata, K. A. Olive, J. Quevillon and J. Zheng, Phys. Rev. D 91, no. 9, 095010 (2015) arXiv:1502.06929 [hep-ph]].

[22] T. D. Brennan, arXiv:1503.08849 [hep-ph].

[23] N. Nagata, K. A. Olive and J. Zheng, JHEP 1510, 193 (2015) arXiv:1509.00809 [hep-ph]].

[24] C. Arbelaez, R. Longas, D. Restrepo and O. Zapata, Phys. Rev. D 93, no. 1, 013012 (2016) arXiv:1509.06313 [hep-ph]]. 
[25] S. M. Boucenna, M. B. Krauss and E. Nardi, Phys. Lett. B 755, 168 (2016) arXiv:1511.02524 [hep-ph]].

[26] J. L. Evans, N. Nagata, K. A. Olive and J. Zheng, JHEP 1602, 120 (2016) arXiv:1512.02184 [hep-ph]].

[27] Y. Mambrini, N. Nagata, K. A. Olive and J. Zheng, Phys. Rev. D 93, no. 11, 111703 (2016) arXiv:1602.05583 [hep-ph]].

[28] M. K. Parida, B. P. Nayak, R. Satpathy and R. L. Awasthi, arXiv:1608.03956 [hepph].

[29] T. W. B. Kibble, G. Lazarides and Q. Shafi, Phys. Lett. B 113, 237 (1982). L. M. Krauss and F. Wilczek, Phys. Rev. Lett. 62, 1221 (1989); L. E. Ibanez and G. G. Ross, Phys. Lett. B 260, 291 (1991); L. E. Ibanez and G. G. Ross, Nucl. Phys. B 368, 3 (1992); S. P. Martin, Phys. Rev. D 46, 2769 (1992) hep-ph/9207218.

[30] H. Georgi and D. V. Nanopoulos, Nucl. Phys. B 159, 16 (1979); C. E. Vayonakis, Phys. Lett. B 82, 224 (1979) [Phys. Lett. 83B, 421 (1979)].

[31] A. Masiero, Phys. Lett. B 93, 295 (1980); Q. Shafi, M. Sondermann and C. Wetterich, Phys. Lett. B 92, 304 (1980); F. del Aguila and L. E. Ibanez, Nucl. Phys. B 177, 60 (1981); R. N. Mohapatra and G. Senjanovic, Phys. Rev. D 27, 1601 (1983); M. Fukugita and T. Yanagida, In *Fukugita, M. (ed.), Suzuki, A. (ed.): Physics and astrophysics of neutrinos* 1-248. and Kyoto Univ. - YITP-K-1050 (93/12,rec.Feb.94) 248 p. C.

[32] M. Fukugita and T. Yanagida, Phys. Lett. B 174, 45 (1986).

[33] H. Georgi, AIP Conf. Proc. 23, 575 (1975); H. Fritzsch and P. Minkowski, Annals Phys. 93, 193 (1975); M. S. Chanowitz, J. R. Ellis and M. K. Gaillard, Nucl. Phys. B 128, 506 (1977); H. Georgi and D. V. Nanopoulos, Nucl. Phys. B 155, 52 (1979).

[34] P. Minkowski, Phys. Lett. B 67 (1977) 421; M. Gell-Mann, P. Ramond and R. Slansky, in Supergravity, eds. D. Freedman and P. Van Nieuwenhuizen (North Holland, Amsterdam, 1979), pp. 315-321. ISBN 044485438x; T. Yanagida, in Proceedings of the Workshop on the Unified Theory and The Baryon Number of the Universe, eds O. Sawada and S. Sugamoto. KEK79-18 (1979); R. N. Mohapatra and G. Senjanovic, 
Phys. Rev. Lett. 44, 912 (1980); J. Schechter and J. W. F. Valle, Phys. Rev. D 22 (1980) 2227; J. Schechter and J. W. F. Valle, Phys. Rev. D 25 (1982) 774.

[35] N. S. Manton, Phys. Rev. D 28, 2019 (1983); F. R. Klinkhamer and N. S. Manton, Phys. Rev. D 30, 2212 (1984); R. F. Dashen, B. Hasslacher and A. Neveu, Phys. Rev. D 10, 4130 (1974).

[36] V. A. Kuzmin, V. A. Rubakov and M. E. Shaposhnikov, Phys. Lett. B 155, 36 (1985).

[37] M. A. Luty, Phys. Rev. D 45, 455 (1992).

[38] N. Cosme, L. Lopez Honorez and M. H. G. Tytgat, Phys. Rev. D 72, 043505 (2005) [hep-ph/0506320]; P. H. Gu, U. Sarkar and X. Zhang, Phys. Rev. D 80, 076003 (2009) arXiv:0906.3103 [hep-ph]]; P. H. Gu and U. Sarkar, Phys. Rev. D 81, 033001 (2010) arXiv:0909.5463 [hep-ph]]; H. An, S. L. Chen, R. N. Mohapatra and Y. Zhang, JHEP 1003, 124 (2010) arXiv:0911.4463 [hep-ph]]; P. H. Gu, Phys. Rev. D 81, 095002 (2010) arXiv:1001.1341 [hep-ph]]; A. Falkowski, J. T. Ruderman and T. Volansky, JHEP 1105, 106 (2011) |arXiv:1101.4936 [hep-ph]]; N. Haba, S. Matsumoto and R. Sato, Phys. Rev. D 84, 055016 (2011) arXiv:1101.5679 [hep-ph]]; E. J. Chun, JHEP 1103, 098 (2011) arXiv:1102.3455 [hep-ph]]; C. Arina and N. Sahu, Nucl. Phys. B 854, 666 (2012) arXiv:1108.3967 [hep-ph]].

[39] J. Shelton and K. M. Zurek, Phys. Rev. D 82, 123512 (2010) arXiv:1008.1997 [hep-ph]]; N. Haba and S. Matsumoto, Prog. Theor. Phys. 125, 1311 (2011) arXiv:1008.2487 [hep-ph]]; W. Z. Feng, A. Mazumdar and P. Nath, Phys. Rev. D 88, no. 3, 036014 (2013) arXiv:1302.0012 [hep-ph]].

[40] T. Gherghetta and G. Jungman, Phys. Rev. D 48, 1546 (1993) hep-ph/9302212.

[41] A.D. Dolgov, and A.D. Linde, Phys. Lett. B116 (1982) 329; D.V. Nanopoulos, K.A. Olive, and M. Srednicki, Phys. Lett. B127 (1983) 30.

[42] J.A. Harvey and M.S. Turner, Phys. Rev. D42 (1990) 3344.

[43] H. K. Dreiner and G. G. Ross, Nucl. Phys. B 410, 188 (1993) hep-ph/9207221.

[44] V.A. Kuzmin, V.A. Rubakov and M.E. Shaposhnikov, Phys. Lett. B191 (1987) 171.

[45] A.E. Nelson and S.M. Barr, Phys. Lett. B246 (1990) 141. 
[46] B. Campbell, S. Davidson, J. Ellis, and K. A. Olive, Phys.Lett. B297 (1992) 118.

[47] S. Davidson, K. Kainulainen and K. A. Olive, Phys. Lett. B 335, 339 (1994) hep$\mathrm{ph} / 9405215$.

[48] M. D'Onofrio, K. Rummukainen and A. Tranberg, Phys. Rev. Lett. 113, no. 14, 141602 (2014) arXiv:1404.3565 [hep-ph]].

[49] M. Fukugita and T. Yanagida, Phys. Rev. D42 (1990) 1285.

[50] B. A. Campbell, S. Davidson, J. R. Ellis and K. A. Olive, Phys. Lett. B 256, 484 (1991); B. A. Campbell, S. Davidson, J. R. Ellis and K. A. Olive, Astropart. Phys. 1, 77 (1992) W. Fischler, G.F. Giudice, R.G. Leigh and S. Paban, Phys. Lett. B258 (1991) 45; L.E. Ibanez and F. Quevedo, Phys. Lett. B283 (1992) 261.

[51] B. A. Campbell, S. Davidson and K. A. Olive, Nucl. Phys. B 399, 111 (1993) hep$\mathrm{ph} / 9302223$.

[52] N. Nagata and S. Shirai, JHEP 1501, 029 (2015) arXiv:1410.4549 [hep-ph]].

[53] N. Nagata and S. Shirai, Phys. Rev. D 91, no. 5, 055035 (2015) arXiv:1411.0752 [hep-ph]].

[54] K. A. Olive and M. Srednicki, Phys. Lett. B 230, 78 (1989); K. A. Olive and M. Srednicki, Nucl. Phys. B 355, 208 (1991); M. Cirelli, A. Strumia and M. Tamburini, Nucl. Phys. B 787, 152 (2007) [arXiv:0706.4071 [hep-ph]].

[55] V. A. Kuzmin and M. E. Shaposhnikov, Phys. Lett. B 92, 115 (1980); T. W. B. Kibble, G. Lazarides and Q. Shafi, Phys. Rev. D 26, 435 (1982); D. Chang, R. N. Mohapatra and M. K. Parida, Phys. Rev. Lett. 52, 1072 (1984); D. Chang, R. N. Mohapatra and M. K. Parida, Phys. Rev. D 30, 1052 (1984); D. Chang, R. N. Mohapatra, J. Gipson, R. E. Marshak and M. K. Parida, Phys. Rev. D 31, 1718 (1985).

[56] M. Miura [Super-Kamiokande Collaboration], arXiv:1610.03597 [hep-ex].

[57] G. Blanger, F. Boudjema, A. Pukhov and A. Semenov, Comput. Phys. Commun. 192, 322 (2015) [arXiv:1407.6129 [hep-ph]].

[58] G. Aad et al. [ATLAS and CMS Collaborations], Phys. Rev. Lett. 114, 191803 (2015) arXiv:1503.07589 [hep-ex]]. 
[59] G. Aad et al. [ATLAS and CMS Collaborations], JHEP 1608, 045 (2016) arXiv:1606.02266 [hep-ex]].

[60] G. Aad et al. [ATLAS Collaboration], JHEP 1511, 206 (2015) arXiv:1509.00672 [hep-ex]].

[61] CMS Collaboration [CMS Collaboration], CMS-PAS-HIG-16-016.

[62] S. D. McDermott, H. B. Yu and K. M. Zurek, Phys. Rev. D 85, 023519 (2012) arXiv:1103.5472 [hep-ph]]; C. Kouvaris and P. Tinyakov, Phys. Rev. Lett. 107, 091301 (2011) arXiv:1104.0382 [astro-ph.CO]].

[63] M. A. Shifman, A. I. Vainshtein and V. I. Zakharov, Phys. Lett. B 78, 443 (1978); A. I. Vainshtein, V. I. Zakharov and M. A. Shifman, Sov. Phys. Usp. 23, 429 (1980) [Usp. Fiz. Nauk 131, 537 (1980)].

[64] A. Abdel-Rehim et al. [ETM Collaboration], Phys. Rev. Lett. 116, no. 25, 252001 (2016) arXiv:1601.01624 [hep-lat]].

[65] J. R. Ellis, K. A. Olive and C. Savage, Phys. Rev. D 77, 065026 (2008) arXiv:0801.3656 [hep-ph]].

[66] B. Borasoy and U. G. Meissner, Annals Phys. 254, 192 (1997) hep-ph/9607432.

[67] D. S. Akerib et al., arXiv:1608.07648 [astro-ph.CO].

[68] E. Aprile et al. [XENON Collaboration], JCAP 1604, no. 04, 027 (2016) arXiv:1512.07501 [physics.ins-det]].

[69] T. Abe, R. Kitano and R. Sato, Phys. Rev. D 91, no. 9, 095004 (2015) arXiv:1411.1335 [hep-ph]].

[70] J. Hisano, K. Ishiwata and N. Nagata, JHEP 1506, 097 (2015) arXiv:1504.00915 [hep-ph]].

[71] R. Mahbubani and L. Senatore, Phys. Rev. D 73, 043510 (2006) hep-ph/0510064; F. D'Eramo, Phys. Rev. D 76, 083522 (2007) arXiv:0705.4493 [hep-ph]]; T. Cohen, J. Kearney, A. Pierce and D. Tucker-Smith, Phys. Rev. D 85, 075003 (2012) [arXiv:1109.2604 [hep-ph]]; C. Cheung and D. Sanford, JCAP 1402, 011 (2014) arXiv:1311.5896 [hep-ph]]; L. Calibbi, A. Mariotti and P. Tziveloglou, JHEP 1510, 
116 (2015) |arXiv:1505.03867 [hep-ph]]. A. Freitas, S. Westhoff and J. Zupan, JHEP 1509, 015 (2015) arXiv:1506.04149 [hep-ph]]; M. A. Fedderke, T. Lin and L. T. Wang, JHEP 1604, 160 (2016) arXiv:1506.05465[hep-ph]]; S. Horiuchi, O. Macias, D. Restrepo, A. Rivera, O. Zapata and H. Silverwood, JCAP 1603, no. 03, 048 (2016) arXiv:1602.04788 [hep-ph]].

[72] S. Banerjee, S. Matsumoto, K. Mukaida and Y. L. S. Tsai, arXiv:1603.07387 [hep-ph].

[73] T. Falk, A. Ferstl and K. A. Olive, Phys. Rev. D 59, 055009 (1999) [Phys. Rev. D 60, 119904 (1999)] hep-ph/9806413]; J. R. Ellis, A. Ferstl and K. A. Olive, Phys. Lett. B 481, 304 (2000) hep-ph/0001005; J. R. Ellis, K. A. Olive, Y. Santoso and V. C. Spanos, Phys. Rev. D 71, 095007 (2005) hep-ph/0502001.

[74] V. Mandic, A. Pierce, P. Gondolo and H. Murayama, hep-ph/0008022; J. R. Ellis, J. L. Feng, A. Ferstl, K. T. Matchev and K. A. Olive, Eur. Phys. J. C 24, 311 (2002) astro-ph/0110225.

[75] C. Cheung, L. J. Hall, D. Pinner and J. T. Ruderman, JHEP 1305, 100 (2013) arXiv:1211.4873 [hep-ph]]; P. Huang and C.E. M. Wagner, Phys. Rev. D 90, no. 1, 015018 (2014) arXiv:1404.0392 [hep-ph]]; A. Crivellin, M. Hoferichter, M. Procura and L. C. Tunstall, JHEP 1507 (2015) 129 arXiv:1503.03478 [hep-ph]].

[76] R. H. Cyburt, J. Ellis, B. D. Fields, F. Luo, K. A. Olive and V. C. Spanos, JCAP 0910, 021 (2009) arXiv:0907.5003 [astro-ph.CO]].

[77] The ATLAS collaboration [ATLAS Collaboration], ATLAS-CONF-2016-050.

[78] CMS Collaboration [CMS Collaboration], CMS-PAS-SUS-16-028.

[79] M. Aaboud et al. [ATLAS Collaboration], Phys. Lett. B 760, 647 (2016) arXiv:1606.05129 [hep-ex]].

[80] V. Khachatryan et al. [CMS Collaboration], arXiv:1609.08382 [hep-ex].

[81] G. Aad et al. [ATLAS Collaboration], Phys. Rev. D 92, no. 1, 012010 (2015) arXiv:1504.03634 [hep-ex]].

[82] N. Nagata, H. Otono and S. Shirai, Phys. Lett. B 748, 24 (2015) arXiv:1504.00504 [hep-ph]]; N. Nagata, H. Otono and S. Shirai, JHEP 1510, 086 (2015) arXiv:1506.08206 [hep-ph]]. 